Aus der Klinik für Kardiologie und Pneumologie

(Prof. Dr. med. G. Hasenfuß)

der Medizinischen Fakultät der Universität Göttingen

\title{
Therapeutische Konsequenzen der Echokardiographie bei Patienten mit ischämischem Schlaganfall
}

\author{
INAUGURAL-DISSERTATION \\ zur Erlangung des Doktorgrades \\ der Medizinischen Fakultät der \\ Georg-August-Universität zu Göttingen \\ vorgelegt von \\ Falko Jonathan Louis, geb. Jürries \\ aus \\ Hamburg
}

Göttingen 2020 
Dekan:

Referent/in:

Ko-Referent/in:

Drittreferent/in:

Datum der mündlichen Prüfung:
Prof. Dr. med. W. Brück

Prof. Dr. med. R. Wachter

Prof. Dr. med. T. R. Döppner

Prof. Dr. med. T. Meyer

05.08.2021 
Hiermit erkläre ich, die Dissertation mit dem Titel "Therapeutische Konsequenzen der Echokardiographie bei Patienten mit ischämischem Schlaganfall" eigenständig angefertigt und keine anderen als die von mir angegebenen Quellen und Hilfsmittel verwendet zu haben.

Göttingen, den 
Die Daten, auf denen die vorliegende Arbeit basiert, wurden teilweise publiziert:

Wachter R, Gröschel K, Gelbrich G, Hamann GF, Kermer P, Liman J, Seegers J, Wasser K, Schulte A, Jürries $F$ et al. (2017): Holter-electrocardiogram-monitoring in patients with acute ischaemic stroke

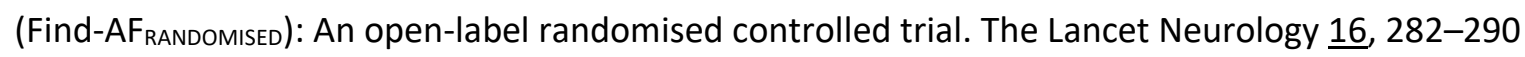
Wasser K, Weber-Krüger M, Jürries F, Liman J, Hamann GF, Kermer P, Uphaus T, Protsenko E, Seegers J, Mende M et al. (2019): The cardiac diagnostic work-up in stroke patients-A subanalysis of the FindAFRANDOMISED trial. PLoS ONE 14 , e021653 


\section{Inhaltsverzeichnis}

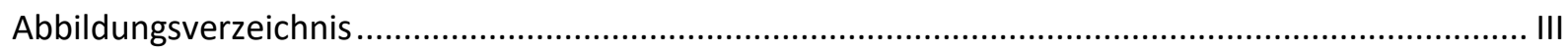

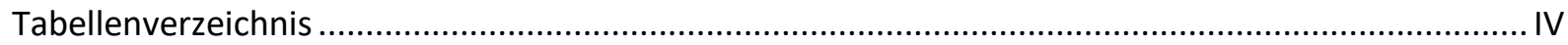

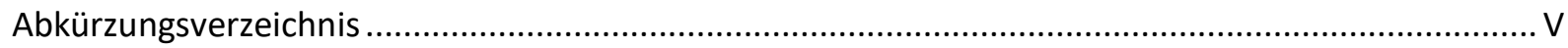

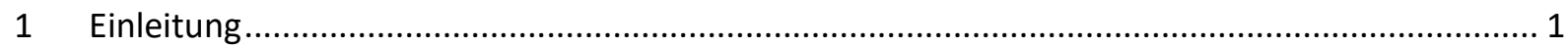

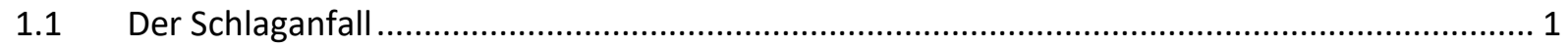

1.1.1 Epidemiologie des Schlaganfalls......................................................................... 1

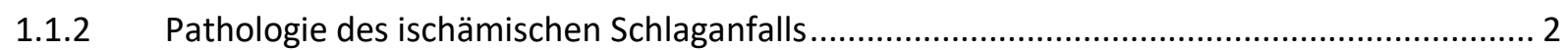

1.1.3 Ätiologische Einteilung des ischämischen Schlaganfalls anhand der TOAST-Ätiologie .......... 3

1.2 Pathophysiologie ausgewählter kardiogener Emboliequellen..............................................6

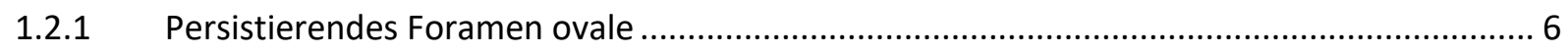

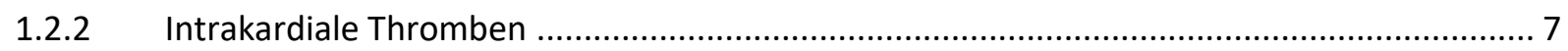

1.3 Diagnostik der kardioembolischen Schlaganfälle und die Rolle der Echokardiographie ........... 7

1.4 Sekundärprävention des kardioembolischen Schlaganfalls und seine Bedeutung .................. 10

1.5 Aktuelle Empfehlungen zum Einsatz der Echokardiographie nach einem ischämischen

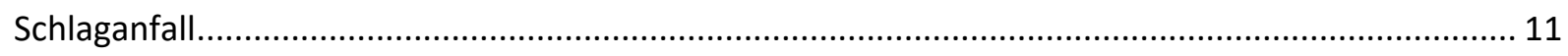

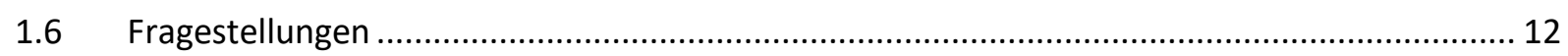

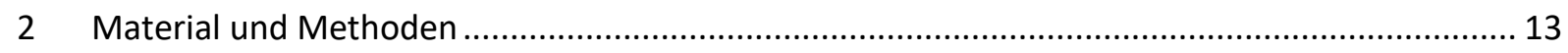

2.1 Studiendesign und Patientenkollektiv .............................................................. 13

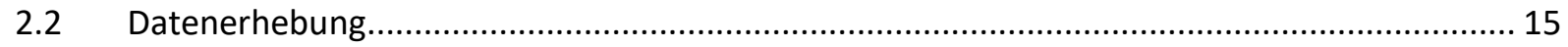

2.2.1 Aufnahme von Daten zur therapeutischen Konsequenz............................................ 17

$2.3 \quad$ Echokardiographische Untersuchung ................................................................. 18

2.4 Erfassung der Daten und statistische Auswertung .................................................... 18

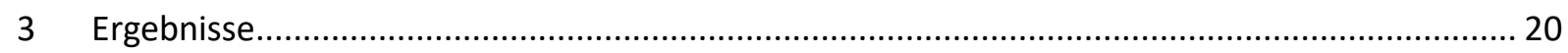

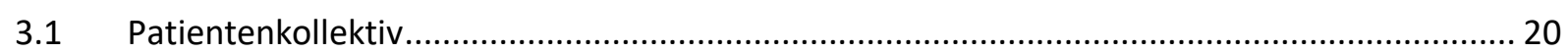

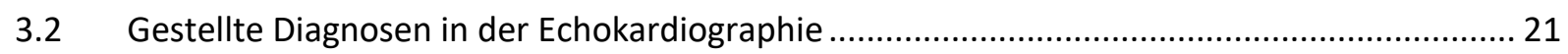

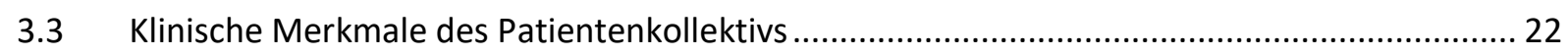

3.4 Therapeutische Konsequenzen durch die Echokardiographie .........................................26

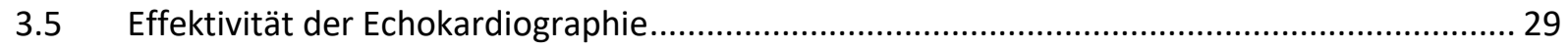

3.5.1 Vergleich der Anzahl therapeutischer Konsequenzen zwischen der Echokardiographie und

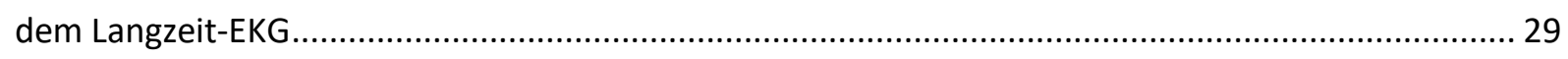

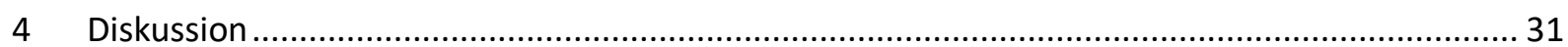




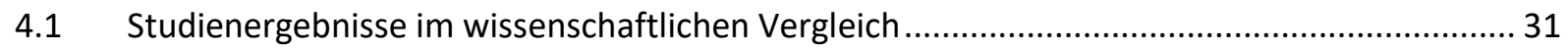

4.2 Bewertung des Patientenkollektivs und der erstellten Patientengruppen.............................. 34

4.3 Bewertung der diagnostischen und therapeutischen Wertigkeit .......................................... 35

4.4 Methodische Einordnung und Limitationen der Studie .......................................................... 40

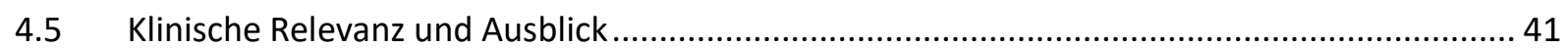

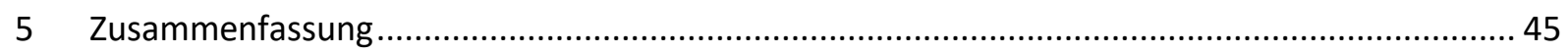

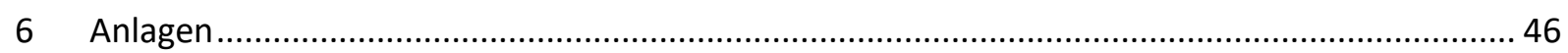

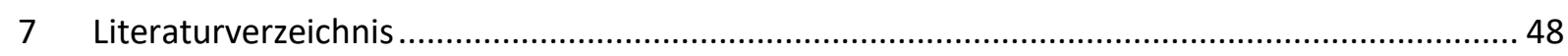




\section{Abbildungsverzeichnis}

Abbildung 1: Studiendesign der Find-AF RANDOMISED-Studie

Abbildung 2: Patientenkollektiv und Verteilung in den randomisierten Gruppen

Abbildung 3: Art und Anzahl der therapeutischen Änderungen durch die Echokardiographie in der

Find-AFrandomised -Studie.

Abbildung 4: Anzahl der gestellten Diagnosen in der Echokardiographie und eingeleiteter therapeutischen Konsequenzen im Vergleich.

Abbildung 5: Vergleich der diagnostischen Methoden anhand der Anzahl therapeutischer Konsequenzen 30

Abbildung 6: Echokardiographische Diagnostik nach ischämischem Schlaganfall 44

Abbildung 7: CRF TTE Untersuchung der Find-AF RANDOMISED -Studie..... 46 Abbildung 8: CRF TEE Untersuchung der Find-AF RANDOMISED-Studie. 


\section{Tabellenverzeichnis}

Tabelle 1: : Übersicht über mögliche kardiale Emboliequellen bei ischämischen Schlaganfall und Einteilung nach Thrombembolie-Risiko .....

Tabelle 2: Übersicht über die Gruppen der TOAST-Klassifikation und deren prozentuale Verteilung. .. 6

Tabelle 3: Übersicht über diagnostizierbare Pathologien mit der Echokardiographie . .9

Tabelle 4: Einschluss und Ausschlusskriterien der Find-AF RANDomISED -Studie....................................... 13

Tabelle 5: Auffällige Befunde in der Echokardiographie ............................................................. 17

Tabelle 6: Anzahl von Pathologien in der Echokardiographie in der Find-AF RANDOMISED -Studie.............. 22

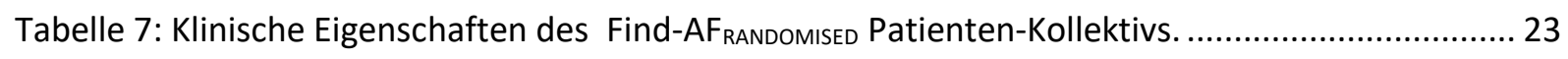

Tabelle 8: Klinische Eigenschaften der Find-AF RANDOMISED -Patienten bei denen kein PFO bestand und Vergleich mit Patienten, bei denen ein PFO diagnostiziert wurde

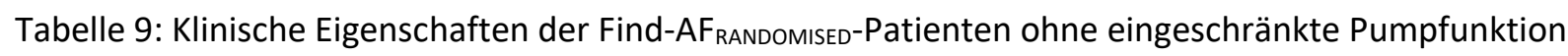
und Vergleich mit Patienten, bei denen eine eingeschränkte Pumpfunktion diagnostiziert wurde.... 25 Tabelle 10: Vergleich der therapeutischen Maßnahmen bei Diagnose eines persistierenden Foramen ovale in den Studienzentren. 


\section{Abkürzungsverzeichnis}

\begin{tabular}{|c|c|}
\hline ASA & Vorhofseptumaneurysma \\
\hline ASS & Acetylsalicylsäure \\
\hline CRF & Case Report Form \\
\hline $\mathrm{CT}$ & Computertomographie \\
\hline CVR & Kardiovaskuläre Risikofaktoren \\
\hline DGN & Deutsche Gesellschaft für Neurologie \\
\hline ECG & Electrocardiography \\
\hline EKG & Elektrokardiogramm \\
\hline ESO & European stroke organization \\
\hline ESUS & Embolic stroke of undetermined source \\
\hline ICD & Implantierbarer Kardioverter-Defibrillator \\
\hline IQR & Interquartilsabstand \\
\hline KHK & Koronare Herzkrankheit \\
\hline LA & Linksatrial \\
\hline LVEF & Linksventrikuläre Ejektionsfraktion \\
\hline MRS & Modifizierte Rankin-Skala \\
\hline MRT & Magnetresonanztomographie \\
\hline $\mathrm{n}$ & Anzahl \\
\hline NASCET & North American Symptomatic Carotid Endarterectomy Trial \\
\hline NIHSS & National Institutes of Health Stroke Scale \\
\hline OAK & Orale Antikoagulation \\
\hline pAVK & periphere arterielle Verschlusskrankheit \\
\hline $\mathrm{PCl}$ & perkutane koronare Intervention \\
\hline PFO & persistierendes Foramen ovale \\
\hline RCT & Randomisierte kontrollierte Studie \\
\hline SD & Standardabweichung \\
\hline TEE & Transösophageale Echokardiographie \\
\hline TIA & Transitorisch ischämische Attacke \\
\hline TOAST-Klassifikation & Trial-of-org-in-acute-stroke-treatment-Klassifikation \\
\hline TTE & Transthorakale Echokardiographie \\
\hline VHF & Vorhofflimmern \\
\hline
\end{tabular}




\section{Einleitung}

Der ischämische Schlaganfall stellt in der westlichen Welt die häufigste Ursache für eine erworbene Behinderung im Alter dar (Heuschmann et al. 2010). Nach einer Datenerhebung der Gmünder Ersatzkasse aus dem Jahr 2005 und 2006 erlitten 11,2\% der Patienten, die einen Schlaganfall erlitten haben, innerhalb von zwölf Monaten ein rezidivierendes ischämisches Ereignis (van den Bussche et al. 2010). Diese Zahlen verdeutlichen die Bedeutung einer gezielten Sekundärprävention. Vorgehensweise, Ablauf und Art der Diagnostik bei Patienten die einen ischämischen Schlaganfall erlitten haben, sind daher wichtiger Gegenstand der aktuellen Forschung.

Mit dem Hintergrund zunehmender Limitierung zeitlicher und finanzieller Ressourcen fällt der Untersuchung möglicher kardioembolischer Ursachen des Schlaganfalls eine besondere Rolle zu, da diese im Vergleich konkurrierender Ätiologien des Hirninfarkts eine höhere Sterblichkeit (Grau et al. 2001) sowie die höchste Gefahr eines Rezidivs aufweisen (Kolominsky-Rabas et al. 1998). Typische Ursachen einer Kardioembolie sind beispielsweise Vorhofflimmern, ventrikuläre Thromben, ein persistierendes Foramen ovale, Klappenvegetationen oder kardiale Tumore (Myxom) (Laufs et al. 2010). Viele dieser Ursachen können mittels Echokardiographie nachgewiesen werden, welche daher einen wichtigen Bestandteil in der ätiologischen Aufarbeitung einer zerebralen Ischämie darstellt. Dabei ist mit dem Hintergrund der Wirtschaftlichkeit und Effizienz neben der Feststellung einer Pathologie durch eine Untersuchung, vielmehr die Auswirkung auf die therapeutische Entscheidung eminent wichtig für den Patienten (Morris et al. 2009).

\subsection{Der Schlaganfall}

\subsubsection{Epidemiologie des Schlaganfalls}

Kardiovaskuläre Erkrankungen sind weltweit gemäß der World Health Organization der häufigste Grund für Todesfälle und Funktionseinschränkungen im täglichen Leben (Mendis et al. 2011). Der Schlaganfall stellt in dieser Gruppe mit weltweit 6.2 Millionen Todesfällen im Jahre 2008 nach dem Herzinfarkt die zweithäufigste Ursache dar (Mendis et al. 2011). Ein weltweiter Anstieg der Inzidenz ist auf Grund der demographischen Entwicklung auch zukünftig zu erwarten und untermauert mit dem Hintergrund der immensen globalen finanziellen Belastung für Therapie 
und Rehabilitation die Wichtigkeit der Primär- und Sekundärprävention dieses Krankheitsbildes (Mendis et al. 2011).

Basierend auf Inzidenzraten des Erlanger Schlaganfall Registers (Kolominsky-Rabas et al. 1998), lag die Inzidenz des Schlaganfalls in Deutschland im Jahr 2008 bei etwa 262.000 (ischämisch und hämorrhagisch), wobei 196.000 einen erstmaligen und 66.000 einen wiederholten Schlaganfall erlitten (Heuschmann et al. 2010). Mit etwa 63.000 Todesfällen ist der Schlaganfall die dritthäufigste Todesursache in Deutschland (Heuschmann et al. 2010). Auch wenn die Mortalitätsraten deutschlandweit in den letzten Jahren einen deutlichen Rückgang zeigen, bleibt der Schlaganfall die häufigste Ursache für erworbene Behinderung im Erwachsenenhalter (Heuschmann et al. 2010). Etwa die Hälfte der Patienten ist auch nach drei Monaten nach dem Ereignis nicht vollständig genesen und viele sind auch weiterhin in ihrem alltäglichen Leben auf externe Hilfe angewiesen (Ward et al. 2005).

\subsubsection{Pathologie des ischämischen Schlaganfalls}

Obwohl das menschliche Gehirn nur zwei Prozent des Körpergewichts ausmacht, verbraucht es etwa $20 \%$ des anfallenden Sauerstoffbedarfs sowie 15\% des Herzzeitvolumens (Eschenfelder et al. 2006). Dieses ist dem Umstand geschuldet, dass das zentrale Nervensystem einen sehr hohen Energiebedarf aufweist, der nur durch die lückenlose Zufuhr von Substraten gedeckt werden kann. Der erforderliche Metabolismus zur Energiegewinnung erfolgt normalerweise aerob durch den Abbau von Glucose (Bähr und Frotscher 2014). Die Substratversorgung erfolgt über den Blutfluss der zum Gehirn führenden Arterien des Karotis-Stromgebietes (Versorgung der vorderen und mittleren Schädelgrube) und des vertebrobasilären Stromgebiets (Versorgung der hinteren Schädelgrube) (Bähr und Frotscher 2014). Der Schlaganfall ist definiert als akut einsetzendes fokales oder globales neurologisches Defizit auf Grund einer umschriebenen Durchblutungsstörung des Gehirns (Eschenfelder et al. 2006). Wird die ständige Zufuhr von Sauerstoff und Glucose zum Gehirn durch einen Gefäßverschluss unterbrochen, kommt es bereits nach wenigen Sekunden zu einem Funktionsverlust der Neurone, da das Gehirn keine Substratvorräte anlegt, um anfallende Unterbrechungen kompensieren zu können (Bähr und Frotscher 2014). Neben dem beschriebenen ischämischen Schlaganfall kommt es in $15-20 \%$ der Fälle zu einer primären Einblutung in das Hirngewebe (Bähr und Frotscher 2014) . In diesem Fall spricht man von einem hämorrhagischen Schlaganfall. Entscheidend für den Patienten ist der Zeitraum der Unterbrechung des Blutflusses, um den Randsaum des ischämischen Kerngebiets, 
auch Penumbra genannt (Astrup et al. 1981), z. B. mit einer systemischen Thrombolyse möglichst schnell der Substratzufuhr wieder anzuschließen, da dieses Gewebe noch keine irreversible Schädigung erlitten hat (Fisher 1997).

Eine wichtige Differentialdiagnose zum ischämischen Schlaganfall besteht in der transitorisch ischämischen Attacke (TIA). Diese ist, im Gegensatz zum Schlaganfall, definiert als zeitlich begrenzte Episode eines fokal neurologischen Ausfalls durch eine zerebrale Ischämie (beispielsweise durch einen zeitlich begrenzten Verschluss einer intrazerebralen Arterie), welche sich jedoch nicht als permanenter Infarkt manifestiert (Easton et al. 2009). Eine veraltete Definition beziffert die Dauer dieser Episode auf maximal 24 Stunden, in neuen Studien konnte jedoch durch diffusions-gewichtete MRT-Bildgebung nachgewiesen werden, dass sich bei 9,8\% dieser Patienten bereits Infarkte passend zur Klinik manifestierten (Coutts et al. 2005). Neuere Definitionen gehen daher von einer Zeitdauer von maximal einer Stunde aus (Albers et al. 2002). Patienten, die eine transitorisch ischämische Attacke erleiden, haben ein hohes Risiko, zeitnah einen Hirninfarkt zu erleiden und sollten daher schnellstmöglich entsprechende Diagnostik zur ätiologischen Abklärung wie MRT-Bildgebung, kardiale Diagnostik oder Ultraschall der hirnversorgenden und intrakraniellen Arterien erhalten (Easton et al. 2009).

\subsection{3 Ätiologische Einteilung des ischämischen Schlaganfalls anhand der TOAST- Ätiologie}

Eine Einteilung der Ursache von Schlaganfällen anhand ihrer Ätiologie ist entscheidend für die Wahl der anschließenden Sekundärprophylaxe und bestimmt somit maßgeblich die Prognose der Patienten. Sie ist daher ein wichtiger Bestandteil in der aktuellen klinischen Aufarbeitung nach einem stattgehabten Schlaganfall. Ein gängiges System zur Einteilung stellt die TOASTKlassifikation dar (Adams et al. 1993). Diese teilt Patienten, welche einen ischämischen Schlaganfall erlitten haben, in fünf Kategorien ein, welche sich auf klinische Befunde, unterstützt durch apparative Diagnostik, begründen. Diese fünf Kategorien sind: Makroangiopathie, kardioembolische Schlaganfälle, Mikroangiopathie, Schlaganfall anderer spezifischer Ursache und Ereignisse multipler und nicht eindeutig zu differenzierender Ursache.

Eine Einteilung der Ursache in die Gruppe der makroangiopathischen Schlaganfälle erfolgt, wenn durch bildgebende Verfahren eine Verengung der hirnversorgenden Gefäße größer als 50\% festgestellt wird. Diese Patienten zeigen gehäuft das klinische Bild einer kortikalen Symptomatik (Aphasien, Neglect) sowie Dysfunktionen des Kleinhirns und Hirnstamms. In den Vorbefunden 
lassen sich häufig Hinweise auf transitorische ischämische Attacken (TIA) finden. Um diese Einteilung vorzunehmen, sollte vorher ein Ausschluss einer möglichen kardioembolischen Ursache des Schlaganfalls durchgeführt werden (Adams et al. 1993).

Ein kardioembolischer Schlaganfall zeigt ein ähnliches Verteilungsmuster sowohl in der zerebralen Bildgebung als auch in der klinischen Präsentation wie die Gruppe der makroangiopathischen Schlaganfälle. Bildmorphologisch zeigen sich typisch ischämische Schlaganfälle in mehr als einem arteriellen Versorgungsgebiet (Adams et al. 1993). Patienten mit einer kardioembolischen Ursache des Schlaganfalls zeigen die höchste Sterblichkeitsrate, das schwerste neurologische Defizit sowie das schlechteste funktionelle Outcome nach 90 Tagen im Vergleich zu anderen Ätiologien (Grau et al. 2001). Grundlage ist der Verschluss eines arteriellen Gefäßes durch einen Embolus aus dem Herzen. Eine mögliche makroangiopathische Ursache sollte vor der Einteilung diagnostisch ausgeschlossen werden (Adams et al. 1993). Pathologien, die als mögliche Ursache eines kardioembolischen Schlaganfalls in Betracht gezogen werden, sind in der Literatur anhand ihres Thrombembolierisikos in zwei Gruppen eingeteilt (Laufs et al. 2010; Ustrell und Pellisé 2010). Eine Übersicht über diese Einteilung gibt Tabelle 1.

Tabelle 1: : Übersicht über mögliche kardiale Emboliequellen bei ischämischen Schlaganfall und Einteilung nach Thrombembolie-Risiko. Tabelle nach Laufs et al. 2010, Ustrell und Pellisé 2010

\begin{tabular}{|l|l|}
\hline Hohes Risiko & Niedriges oder unklares Risiko \\
\hline Vorhofflimmern & Persistierendes Foramen ovale \\
Vorhofflattern & Vorhofseptumaneurysma \\
Sick sinus syndrome & $\begin{array}{l}\text { Spontaner Echokontrast im linken Vorhof } \\
\text { (Smoke) }\end{array}$ \\
Linksatrialer Thrombus & Mitralklappenprolaps \\
Mitralklappenstenose & Mitralringverkalkung \\
Mechanischer Klappenersatz & Sklerosiende Aortenklappenstenose \\
Linksventrikulärer Thrombus & Wandbewegungsstörungen des linken \\
Kürzlich stattgefundener Herzinfarkt & Ventrikels \\
Dilitative Kardiomyophathie & \\
Endokarditis & \\
Komplexe Atheromatose des Aortenbogens & \\
Vorhofmyxom & \\
\hline
\end{tabular}

Dabei muss berücksichtigt werden, dass für einige dieser aufgezählten Befunde eine klare Assoziation mit einem ischämischen Schlaganfall bisher noch nicht bewiesen werden konnte (Laufs et al. 2010). Bei einigen Diagnosen, wie beispielweise der in der Tabelle aufgeführte spontane Echokontrast im linken Vorhof, ist noch unklar, ob diese mit dem Schlaganfall oder 
doch mit einer Begleiterkrankung, wie in diesem Fall dem Vorhofflimmern, assoziiert werden sollte, was auch die Entwicklung einer therapeutischen Strategie erschwert (Yang et al. 2016).

Patienten mit Schlaganfällen auf Grundlage mikroangiopathischer Veränderungen weisen klinisch klassische lakunäre Syndrome nach und keine kortikalen oder zerebellären Dysfunkionen. Klassische lakunäre Syndrome manifestieren sich als rein motorische Hemiparesen, rein sensible Hemihypästhesien oder ataktische Hemiparesen (Arboix and Martí-Vilalta 2009). Bildgebende Verfahren wie CT oder MRT zeigen entweder keine oder nur kleine subkortikale Läsionen. Unterstützt wird die Einteilung durch Vorerkrankungen aus dem Spektrum der kardiovaskulären Risikofaktoren wie Diabetes Mellitus oder arterielle Hypertension (Adams et al. 1993: 36).

Schlaganfälle anderer spezifischer Ursache werden beschrieben, wenn seltene Krankheiten wie beispielsweise Hyperkoagulopathien als Auslöser des ischämischen Schlaganfalls nachgewiesen werden können. In der letzten Gruppe der Ereignisse unbekannter Ursache werden Schlaganfälle klassifiziert, bei denen kein zugrundeliegender Auslöser mit Sicherheit identifiziert werden kann. Weiterhin fallen auch Patienten in diese Kategorie, bei denen sich mehrere mögliche Auslöser eines Schlaganfalls akkumulieren (Adams et al. 1993: 36).

Diese zuletzt genannte Kategorie der Ereignisse unbekannter Ursache, ist auf Grund der hohen Inzidenz ein wichtiger Bestandteil heutiger Forschung, da sich für diese hohe Anzahl an Patienten die unbefriedigende Situation ergibt, keine Ursache für den stattgefundenen Schlaganfall zu identifizieren und somit auch ein Ansatz für eine spezifische Therapie fehlt. Ein möglicher Ansatzpunkt entwickelte sich in den letzten Jahren durch die ESUS Klassifikation, welche postuliert, dass Schlaganfälle unbekannter Ursache in der Mehrzahl der Fälle thrombembolischer Genese sind und diese Patientengruppe von einer oralen Antikoagulation als Sekundärprophylaxe im Gegensatz zu einer Thrombozytenaggregationshemmung profitieren würde (Hart et al. 2014). Die Diagnose eines ESUS wird nach folgenden Kriterien gestellt: Bildgebender Nachweis (CT oder MRT) eines nicht lakunären Hirninfarkts, Ausschluss einer relevanten Makroangiopathie mit konsekutiver Einengung von 50\% der das Ischämiearreal versorgenden Arterie, die Abwesenheit konkurrierender Hoch-Risiko- Quellen einer Thrombembolie oder verminderte Ejektionsfraktion des linken Ventrikels $<30 \%$, sowie fehlende andere spezifische Gründe eines Hirninfarkts (z. B. eine zerebrale Vaskulitis, Dissektionen, Vasospasmen, Migräne, Substanz-Missbrauch) (Hart et al. 2014).

Eine Übersicht über die verschiedenen Gruppen der Toast Klassifikation und ihre Häufigkeit zeigt Tabelle 2. 
Tabelle 2: Übersicht über die Gruppen der TOAST-Klassifikation und deren prozentuale Verteilung. Tabelle nach Ringelstein und Nabavi 2007, Zahlen zur Inzidenz Kolominsky-Rabas et al. 2001

\begin{tabular}{|c|c|c|}
\hline TOAST Gruppe & TOAST-Kriterien & $\begin{array}{l}\text { Inzidenz (pro } \\
\text { 100.000), } \\
\text { standardisiert } \\
\text { für europäische } \\
\text { Population }\end{array}$ \\
\hline $\begin{array}{l}\text { 1. Arteriosklerose der } \\
\text { großen } \\
\text { hirnversorgenden } \\
\text { Arterien (zerebrale } \\
\text { Makroangiophathie) }\end{array}$ & $\begin{array}{l}\text { - Symptomatik oder Infarkt auf ein } \\
\text { - } \quad \text { Infarkt kortitorium beschränkt } \\
1,5 \mathrm{~cm} \text {. } \\
\text { - } \quad \text { Stenose }>50 \% \text { der der extra oder } \\
\text { intrakraniellen versorgenden Arterien } \\
\text { - } \quad \text { Ausschluss anderer Ätiologien }\end{array}$ & 15,3 \\
\hline 2. Kardiogene Embolie & $\begin{array}{l}\text { - Klinik passend zu thrombembolischem } \\
\text { Insult } \\
\text { - Infarkte in mehreren Gefäßterritorien oder } \\
\text { Infarkt rein kortikal oder } \\
\text { Infarktdurchmesser }>1,5 \mathrm{~cm} \\
\text { - Signifikante kardiale Emboliequelle } \\
\text { - } \quad \text { Ausschluss anderer Emboliequellen }\end{array}$ & 30,2 \\
\hline $\begin{array}{l}\text { 3. Zerebrale } \\
\text { Mikroangiopathie }\end{array}$ & $\begin{array}{l}\text { - Symptomatik passend zu lakunärem Insult } \\
\text { - Infarkt subkortikal und Infarktdurchmesser } \\
\quad<1,5 \mathrm{~cm} \\
\text { - } \quad \text { Ausschluss anderer Ätiologien }\end{array}$ & 25,8 \\
\hline 4. Andere Ätiologie & $\begin{aligned} & \text { - Auswahl } \\
& \circ \text { Gefäßdissektion } \\
& \circ \text { Zerebrale Vaskulitis } \\
& \circ \text { Thrombophilie } \\
& \circ \text { Mitochondriopathie } \\
& \circ \text { latrogen }\end{aligned}$ & 2,1 \\
\hline 5. Unklare Ätiologie & $\begin{array}{l}\text { - Keine potentielle Ätiologie oder } \\
\text { - } \quad>1 \text { potentielle Ätiologie oder } \\
\text { - Unvollständige Diagnostik }\end{array}$ & 39,3 \\
\hline
\end{tabular}

\subsection{Pathophysiologie ausgewählter kardiogener Emboliequellen}

\subsubsection{Persistierendes Foramen ovale}

Das persistierende Foramen ovale (PFO) ist der häufigste angeborene Herzfehler fetaler Abstammung und lässt sich bei etwa $25 \%$ der erwachsenen Bevölkerung nachweisen (Homma et al. 2016). Es resultiert aus dem fehlenden Verschluss des physiologisch vorkommenden Foramen ovale, welches während der Fetalzeit eine direkte Verbindung zwischen dem rechten und linken Vorhof zur Umgehung des Lungenkreislaufes darstellt (Homma et al. 2016). Auslöser des Schlaganfalls ist eine paradoxe Embolie, bei welcher Thromben aus dem venösen Stromgebiet, 
beispielsweise im Rahmen einer tiefen Beinvenenthrombose, durch das Foramen ovale in den arteriellen Kreislauf gelangen (Desai et al. 2006). Auf Grund seines gehäuften Auftretens bei Patienten, die einen Schlaganfall unbekannter Ursache erlitten haben, gilt das Vorhandensein eines persistierenden Foramen ovale als Risikofaktor zum Erleiden eines Schlaganfalls (Lechat et al. 1988).

\subsubsection{Intrakardiale Thromben}

Intrakardiale Thromben entstehen in unterschiedlichen anatomischen Strukturen des Herzens. Zum einen im Vorhof oder Vorhofohr (LA/LAA Thrombus), welche häufig bei Patienten mit einem Vorhofflimmern beobachtet werden können (Di Minno et al. 2016). Pathophysiologisch unterliegt die Entstehung der Thromben vor allem den Gesetzmäßigkeiten der Virchowschen Trias, welche besagt, dass die Bildung von Thromben durch Veränderungen der Gefäßwand, der Blutströmung und der Zusammensetzung des Bluts bedingt ist (Haas 2005). Dem Blutfluss folgend, gelangen diese Thromben in das arterielle System und verursachen durch den Verschluss einer extra- oder intrakraniellen Arterie einen Hirninfarkt.

Beim Vorhofflimmern kommt es auf Grund einer verringerten Kontraktilität und Vergrößerung des linken Vorhofs zu einer Verlangsamung der Flussgeschwindigkeit (Iwasaki et al. 2011), wodurch ein hohes Risiko zur lokalen Bildung von Thromben entsteht. Zusätzlich wird die Thrombogenität bei Patienten mit einem Vorhofflimmern durch Ausschüttung des vonWillebrand-Faktors, einem Marker für eine Schädigung des Endothels (Conway et al. 2003), erhöht.

Linksventrikuläre Thromben hingegen lassen sich ätiologisch häufig auf einen Herzinfarkt (insbesondere Vorderwandinfarkte) oder eine dilitative Kardiomyopathie zurückführen (Stokman et al. 2001). Pathophysiologisch unterliegt die lokale Entstehung des Thrombus ebenfalls den Gesetzmäßigkeiten der Virchowschen Trias (Stokman et al. 2001).

\subsection{Diagnostik der kardioembolischen Schlaganfälle und die Rolle der Echokardiographie}

Pathologien, welche die Ursache für einen kardioembolischen Schlaganfall sein könnten, werden in der klinischen Diagnostik durch verschiedene Mittel erfasst. So spielt das EKG die entscheidende Rolle in der Diagnostik des Vorhofflimmerns, der häufigsten Ursache des kardioembolischen Schlaganfalls (Ustrell und Pellisé 2010). Die Frage, ob das permanente 
Vorhofflimmern das höchste assoziierte Risiko hat, einen Schlaganfall auszulösen (Vanassche et al. 2015) oder die persistierende und permanente Form der paroxysmalen in dieser Hinsicht gleichzusetzen ist (Hohnloser et al. 2007), ist nicht abschließend beantwortet.

Die Diagnostik eines möglicherweise vorliegenden Vorhofflimmerns erfolgt über ein standartmäßiges 12-Kanal EKG oder eine Langzeit-EKG Untersuchung. Die zum Einsatz kommenden Holter-EKGs mit einer Tragezeit von bis zu zehn Tagen sind eine effektive Methode zur Detektion eines zugrundeliegenden paroxysmalen Vorhofflimmerns (Wachter et al. 2017). Neben den tragbaren Langzeit-EKGs zeigte sich, dass Eventrecorder, zum einen implantiert (Sanna et al. 2014; Cotter et al. 2013) oder extern (Gladstone et al. 2014) appliziert, ebenfalls als diagnostische Methode eine Alternative zur Detektion von paroxysmalen Vorhofflimmern darstellen.

Einen weiteren wichtigen Bestandteil in der Diagnostik möglicher kardialer Emboliequellen stellt die Echokardiographie dar, welche im Fokus dieser Arbeit steht. Die Deutsche-SchlaganfallGesellschaft empfiehlt in Ihren Zertifizierungskriterien für Stroke Units mindestens 15\% aller Patienten, die einen ischämischen Hirninfarkt erlitten haben, mittels transösphagealer Echokardiographie zu untersuchen (Nabavi et al. 2015). Ziel dieser Untersuchung ist es, Quellen möglicher kardialer Embolien anhand struktureller Anomalien zu identifizieren.

Die Echokardiographie kann in zwei verschiedenen Formen durchgeführt werden. Zum einen gibt es die transthorakale (TTE), zum anderen die transösophageale Echokardiographie (TEE). Die transthorakale Echokardiographie wird durch eine von außen auf der Brustwand positionierte Sonde durchgeführt, die transösophageale Echokardiographie hingegen durch eine Schlucksonde, welche durch ein Endoskop herznah im Ösophagus platziert wird. Die thransthorakale Echokardiographie zeichnet sich dadurch aus, dass sie weit verbreitet, nicht invasiv, günstiger und einfacher durchzuführen ist (Morris et al. 2009). Jedoch ist die Sensitivität zur Erkennung möglicher kardioembolischer Quellen geringer (Morris et al. 2009).

Dabei zeigt sich, dass die transthorakale Echokardiographie unter normal einzuordnenden Schallbedingungen die überlegenere Methode zur Feststellung linksventrikulärer Thromben, linksventrikulärer Hypertrophien, Wandbewegungsstörungen, Mitral- und Aortenklappenstenosen sowie Mitralringverkalkungen darstellt und daher auch unter Berücksichtigung der Risiken einer transösophagealen Echokardiographie bei dieser Fragestellung die geeignetere Methode darstellt (Busse und Darius 2002). Bei bestehendem Verdacht auf eine infektiöse Endokarditis als Emboliequelle sollte die transthorakale Echokardiographie zwar bei jedem Patienten durchgeführt werden, ist aber der Sensitivität der transösophagealen Variante 
unterlegen (Pepi et al. 2010). Einen weiteren Vorteil bietet die transthorakale Echokardiographie bei der Entdeckung bisher unbekannter kardiovaskulärer Erkrankungen (Herzinfarkt, Koronare Herzkrankheit, Herzinsuffizienz, arterielle Hypertonie, Klappenannomalien), da diese ähnliche Risikofaktoren mit dem Schlaganfall teilen und seine Prognose dadurch bestimmen (Laufs et al. 2010). Bevorstehende Manifestationen und Komplikationen können so durch die Einleitung einer präventiven Therapie verhindert werden (Laufs et al. 2010).

Die transösophageale Echokardiographie hingegen stellt eine invasive Maßnahme dar und ist mit höheren Kosten und erhöhtem Aufwand verbunden, zeigt jedoch hinsichtlich der Beurteilung von Vorhofthromben, Vorhofseptumdefekten (z. B. persistierendes Foramen ovale), Vorhofseptumaneurysma, Klappenvegetationen, spontane Echokontrastbildung, Aortenbogenplaques, Mitralklappenprolaps, Vorhofmyxome und zur Beurteilung von künstlichen Herzklappen (Busse und Darius 2002) eine höhere diagnostische Wertigkeit im Vergleich zur transthorakalen Untersuchung (Morris et al. 2009). Auch wenn die transösophageale Echokardiographie gerade in den Händen eines routinierten Untersuchers eine sehr sichere invasive Methode darstellt (Peterson et al. 2003; Daniel et al. 1991), bleibt ein geringes Risiko für Komplikationen wie Läsionen im Mund- und Rachenraum, Herzrhythmusstörungen oder gastrointestinale Blutungen bestehen (Lambertz und Lethen 2013).

Eine Übersicht über die potentiellen Pathologien, die als Emboliequelle in Frage kommen und mit welcher Untersuchungsmethode diese bevorzugt diagnostiziert werden sollten, zeigt Tabelle 3.

Tabelle 3: Übersicht über diagnostizierbare Pathologien mit der Echokardiographie nach Busse und Darius 2002

\begin{tabular}{l|l}
\hline TTE & TEE \\
\hline $\begin{array}{l}\text { Linksventrikulärer Thrombus } \\
\text { Linksventrikuläre Hypertrophie }\end{array}$ & $\begin{array}{l}\text { Vorhof oder Vorhofohrthrombus } \\
\text { Linksventrikulärer Spitzenthrombus } \\
\text { Wandbewegungsstörungen, Dyskinesien }\end{array}$ \\
$\begin{array}{l}\text { Mitral und Aortenklappenstenose } \\
\text { Eventuell Mitralringverkalkung }\end{array}$ & $\begin{array}{l}\text { Vorhofseptumaneurysma } \\
\text { Klappenvegetationen }\end{array}$ \\
& $\begin{array}{l}\text { Fehlfunktionen künstlicher Herzklappen } \\
\text { Spontane Echokontrastbildung im linken Vorhof }\end{array}$ \\
& $\begin{array}{l}\text { Aortenbogenplaques und -thromben } \\
\text { Mitralklappenprolaps }\end{array}$ \\
& Vorhofmyxom
\end{tabular}

TEE: Transösophageale Echokardiograpghie; TTE: Transthorakale Echokardiographie 


\subsection{Sekundärprävention des kardioembolischen Schlaganfalls und seine Bedeutung}

Im klinischen Alltag sollte es das Ziel sein, die Einstellung einer effektiven und leitliniengerechten Sekundärprophylaxe sicherzustellen, welche die Wahrscheinlichkeit weiterer ischämischer Ereignisse verringert. Wie bereits erwähnt, kommt der Gruppe der kardioembolischen Schlaganfälle eine besondere Bedeutung zu. Untermauert wird dies durch die hohe Wahrscheinlichkeit von 22\% in zwei Jahren, einen weiteren embolischen Hirninfarkt zu erleiden. Zudem besteht bei Diagnose einer kardialen Emboliequelle die niedrigste Zwei-JahresÜberlebenszeit nach einem Erstereignis im Vergleich zu den konkurrierenden Ätiologien der TOAST-Klassifikation (Kolominsky-Rabas et al. 2001). Die Emboli, welche aus dem Herzen stammen und einen Schlaganfall verursachen, sind häufig groß, führen bei den betroffenen Personen vermehrt zu bleibenden Behinderungen und zeigen hohe Sterberaten (Arboix and Alió 2010). Unter diesem Gesichtspunkt ist es besonders wichtig bei Patienten, die möglicherweise einen kardioembolischen Schlaganfall erlitten haben, eine Quelle des Embolus zu identifizieren. Als Konsequenz ist, je nach diagnostizierter Pathologie, ein angepasstes therapeutisches Management mit einem spezifischen Therapieansatz (Yang et al. 2016) im Gegensatz zur standardisierten Sekundärprophylaxe nach erlittenem Schlaganfall (DGN 2015) notwendig.

So senkt die Einstellung auf eine orale Antikoagulation bei Patienten mit einem Vorhofflimmern, unabhängig davon ob es in der permanenten, persistierenden oder paroxysmalen Form besteht, deutlich das Risiko, einen weiteren Schlaganfall zu erleiden (Hart et al. 2003). Auch profitieren Patienten mit einem linksventrikulären Thrombus als Emboliequelle von einem Beginn einer oralen Antikoagulation (Vaitkus und Barnathan 1993). Im Falle einer Diagnose eines persistierenden Foramen ovale besteht nach aktuellen Leitlinien nur für Patienten im Alter von 16-60 Jahren, welche einen kryptogenen Schlaganfall erlitten, als auch einen mittelgroßen bis großen Rechts-Links-Shunt aufweisen, eine Empfehlung zum interventionellen Verschluss mittels Disc-Okkluder (Diener et al. 2018). Ob Patienten, die ein Alter > 60 Jahre aufweisen von einem Verschluss profitieren, kann auf Grund mangelnder Daten derzeit nicht beantwortet werden (Diener et al. 2018). In der medikamentösen Sekundärprophylaxe im Falle eines diagnostizierten Foramen ovale zeigt sich keine Überlegenheit einer oralen Antikoagulation im Vergleich zur Behandlung mit einer Thrombozythenaggregationshemmung (Furlan et al. 2012; Mas et al. 2016) bezüglich der Risikoreduktion erneuter ischämischer Ereignisse. Daher wird im Fall einer Ablehnung eines interventionellen Verschlusses bei Patienten $<60$ Jahre mit einem kryptogenen Schlaganfall und einem offenen Foramen ovale eine Sekundärprophylaxe mit Aspirin oder Clopdiogrel empfohlen (Diener et al. 2018). Der Einsatz einer oralen Antikoagulation bei 
Patienten, welche eine Kombination eines Foramen ovale und rezidivierenden venösen Thrombosen, Lungenembolien oder Koagulationsstörungen aufweisen, bleibt auf Grund der mangelhaften Datenlage (Wechsler 2008) eine Off-Label-Indikation.

Da die orale Antikoagulation mit einem erhöhten Risiko für Blutungen in verschiedene Organsysteme einhergeht, sollte eine Indikationsstellung nicht leichtfertig erfolgen. Nach aktuellen deutschen Leitlinien empfiehlt die deutsche Gesellschaft für Neurologie (DGN) den Einsatz einer oralen Antikoagulation bei Vorliegen von nicht-valvulärem Vorhofflimmern zur Sekundärprophylaxe (DGN 2015). Zudem wird eine orale Antikoagulation bei mechanischen Klappenprothesen, Mitralstenosen (in Erwägung zu ziehen bei zusätzlich vorliegenden spontanen Echokontrast oder vergrößerten Vorhof) und linksventrikulären Thromben empfohlen (Ponikowski et al. 2016; Zeymer et al. 2013; Vahanian et al. 2012).

\subsection{Aktuelle Empfehlungen zum Einsatz der Echokardiographie nach einem ischämischen Schlaganfall}

Bisher existiert keine ausreichende Datenlage über den optimalen Einsatz für eine echokardiographische Untersuchung bei Patienten mit einem ischämischen Schlaganfall (Ustrell und Pellisé 2010). In Deutschland wurde daher weder in der Kardiologie noch in der Neurologie eine klare Leitlinie verfasst, ob und bei welchen Patienten eine Echokardiographie durchgeführt werden sollte (DGN 2017). So empfiehlt die Deutsche Gesellschaft für Neurologie: „Bei Patienten mit Hirninfarkt oder TIA ist die Durchführung einer Echokardiografie (TTE/TEE) sinnvoll, insbesondere wenn keine eindeutige Schlaganfallursache vorliegt." (DGN 2017). Die European Stroke Organisation (ESO) empfiehlt den Einsatz der Echokardiographie nur bei ausgewählten Patienten (Class III, Level B) (Ringleb et al. 2008), die American Stroke Association erwähnt keine klare Empfehlung zum Einsatz in ihrer Leitlinie (Kernan et al. 2014).

Eine Empfehlung zur Indikation für die transösophageale Echokardiographie wird kontrovers diskutiert. Mit dem Hintergrund, dass es sich um eine invasive Methode handelt, sollte eine Indikation nur gestellt werden, wenn Erkenntnisse zu therapeutischen Konsequenzen führen (Laufs et al. 2010: 618). Andererseits wird argumentiert, dass es durch einen breiten Einsatz in der Patientengruppe der kryptogenen Schlaganfälle zur erhöhten Indikationsstellung einer oralen Antikoagulation als Sekundärprophylaxe kommt (Harloff et al. 2006). Die Deutsche Gesellschaft für Neurologie empfiehlt den Einsatz im Falle einer unklaren Ursache des Schlaganfalls bei 
hochgradigem Verdacht auf eine kardiale Emboliequelle oder Planung möglicher interventioneller Eingriffe (z. B. Vorhofohrverschluss) (DGN 2017).

\subsection{Fragestellungen}

Konkret lassen sich daraus folgende Fragen ableiten:

1.) Profitieren Patienten nach einem erlittenen ischämischen Schlaganfall durch einen Einsatz der Echokardiographie als Methode zur ätiologischen Abklärung und welche diagnostischen Erkenntnisse können gewonnen werden?

2.) Wie hoch ist die klinische Bedeutung der Echokardiographie anhand der Häufigkeit einer Änderung des therapeutischen Managements des Patienten einzuschätzen?

3.) Bestehen Unterschiede in der Wertigkeit der transthorakalen gegenüber der transösophagealen Echokardiographie?

4.) Wie ist die klinische Wertigkeit der Echokardiographie im Vergleich mit anderen diagnostischen Methoden wie einer Langzeit-EKG-Untersuchung durch Holter Monitoring zu beurteilen? 


\section{Material und Methoden}

\subsection{Studiendesign und Patientenkollektiv}

Bei der Verfassung dieser Arbeit wurden Daten verwendet, welche im Rahmen der Find-AFRANDomised -Studie erhoben wurden (RCT Nummer: NCT01855035). Find-AFrandomised ist eine randomisierte und kontrollierte, prospektive, multizentrische Studie. Zur Erhebung der Daten wurden an vier Studien-Zentren (Klinik für Neurologie der Universitätsmedizin Göttingen, Klinik und Poliklinik für Neurologie der Universitätsmedizin Mainz, Klinik für Neurologie des NordwestKrankenhaus Sanderbusch, Klinik für Neurologie der Helios Dr. Horst Schmidt Kliniken Wiesbaden), im Zeitraum von Mai 2013 bis August 2014 insgesamt 402 Patienten eingeschlossen. Im Zentrum Göttingen erfolgte der Einschluss der Patienten durch die Klinik der Kardiologie und Pneumologie in Zusammenarbeit mit den Kollegen der Neurologie auf der zertifizierten Stroke Unit. Patienten mit einem Alter von über 60 Jahren, welche mit Symptomen eines Schlaganfalls auf die Stroke Unit aufgenommen wurden, sind anschließend durch das Studienpersonal in die Screening Liste aufgenommen worden. Zum möglichen Einschluss in die Studie mussten die Patienten die folgenden Voraussetzungen erfüllen:

Tabelle 4: Einschluss und Ausschlusskriterien der Find-AF RANDOMISED -Studie

\begin{tabular}{|c|c|}
\hline Einschlusskriterien & Ausschlusskriterien \\
\hline 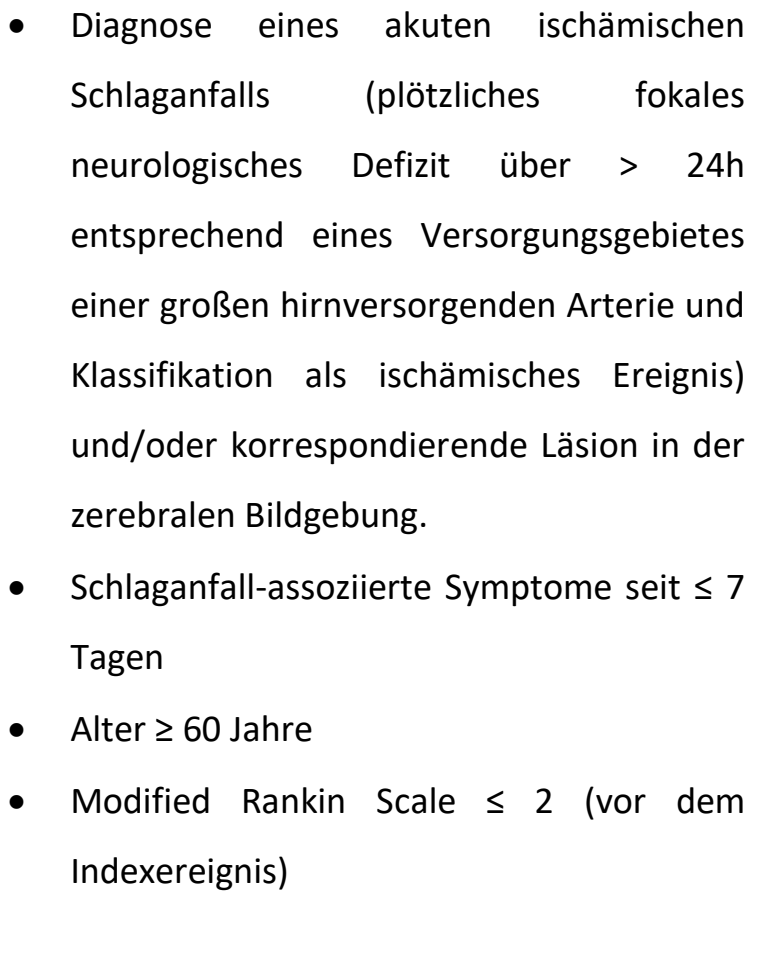 & 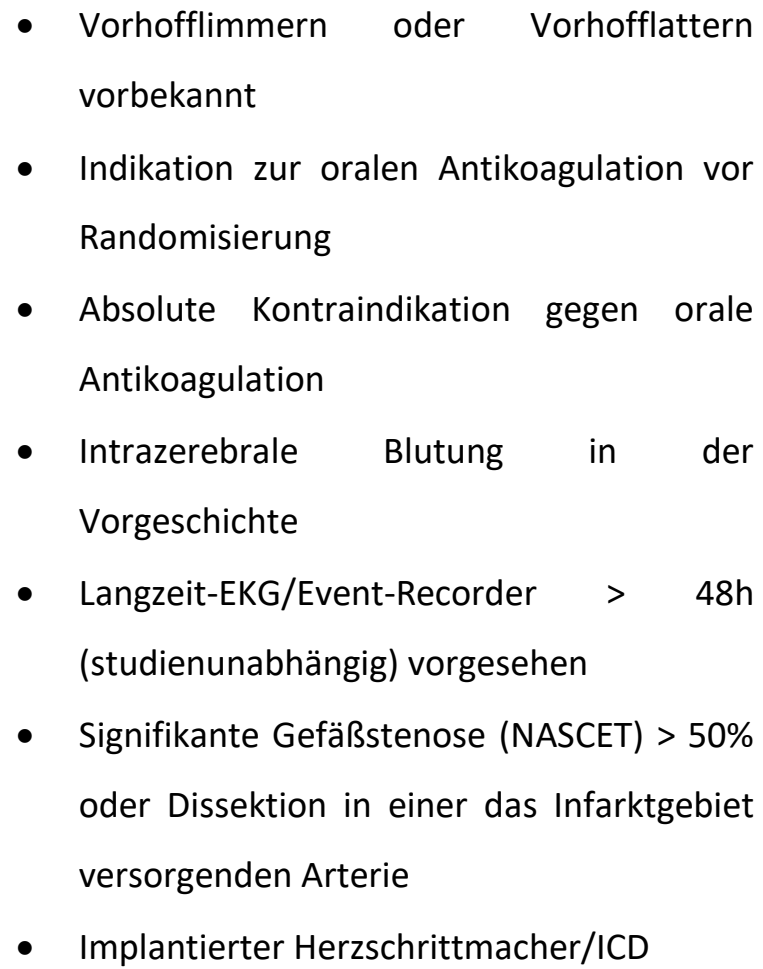 \\
\hline
\end{tabular}




\begin{tabular}{|l|l|}
\hline & $\begin{array}{l}- \text { Lebenserwartung }<1 \text { Jahr (z. B. aufgrund } \\
\text { metastasierten Krebsleidens) } \\
\bullet\end{array}$ \\
Teilnahme an einer anderen \\
randomisierten kontrollierten Studie
\end{tabular}

Nach einer Aufklärung und dem Einholen des schriftlichen Einverständnisses durch die Studienärzte wurden die Patienten gleichermaßen (Verhältnis 1:1, balancierter Randomisierungsmodus) in zwei Gruppen randomisiert. Patienten der Untersuchungsgruppe A erhielten zusätzlich zur leitliniengerechten Schlaganfall-Diagnostik eine über 10 Tage andauernde, zu drei Zeitpunkten (bei Aufnahme, nach drei und nach sechs Monaten) abgeleitete Langzeit-EKGÜberwachung, welche zentral in einem EKG-Labor nach präspezifiziertem Schema ausgewertet wurde (,enhanced and prolonged ECG-monitoring. “). Die Kontrollgruppe erhielt eine den aktuellen Leitlinien entsprechende Diagnostik entsprechend dem lokalen Klinik-Standart („usual care", mindestens 24 Stunden EKG-Überwachung). Um eine weiterführende klinische Beobachtung zu ermöglichen, sah das Studienprotokoll an drei festgelegten Terminen (V1 nach 3 Monaten, V2 nach 6 Monaten, V3 nach 12 Monaten) eine ambulante Nachuntersuchung für alle Patienten vor. Das Studiendesign ist in Abbildung 1 dargestellt.

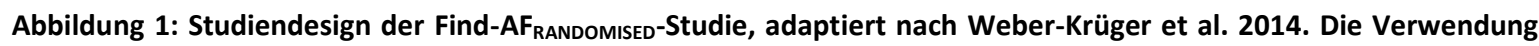
erfolgt mit freundlicher Genehmigung vom Elsevier Verlag
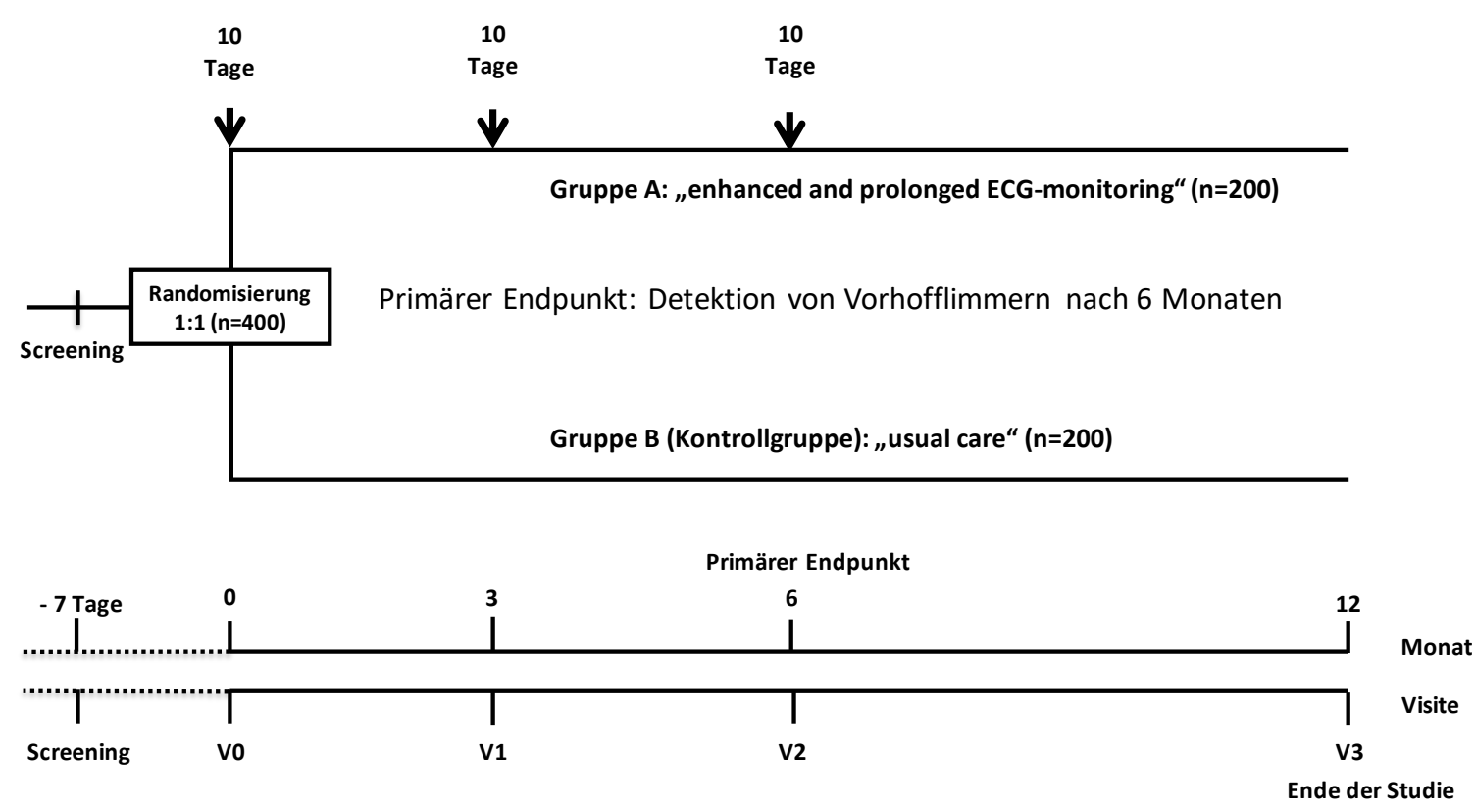
Bei nicht vorhandener Zustimmungsfähigkeit seitens des Patienten wurde nach Absprache mit Verwandten ersten Grades eine mutmaßliche Teilnahme an der Studie zugelassen. Das Einverständnis durch den Patienten konnte zu einem späteren Zeitpunkt nachgeholt werden.

Primärer Endpunkt der Find-AF $F_{\text {RANDOMISED }}$-Studie war, ein neu aufgetretenes Vorhofflimmern oderflattern (> 30 Sekunden) vor Auftreten eines weiteren Schlaganfalls innerhalb von sechs Monaten nach Studieneinschluss nachzuweisen. Die Auswertung und Analyse der Langzeit-EKG-Daten erfolgte zentral im Studienzentrum der Universitätsmedizin Göttingen, zum einen automatisch durch eine Software, zum anderen jedoch manuell, beispielsweise in Phasen mit einer hohen Frequenz an Artefakten. Alle für eine Neudiagnose eines Vorhofflimmerns in Frage kommenden Rhythmusereignisse auf der einen wie auch mögliche neu aufgetretene zerebrale Ischämien auf der anderen Seite wurden durch externe, unabhängige, gegenüber anderen Daten verblindete Adjudikations-Komitees überprüft und evaluiert. Ein neu aufgetretenes Vorhofflimmern wurde nach den aktuell gültigen Leitlinien diagnostiziert (Camm et al. 2010).

Vor Einschluss des ersten Patienten lag das Ethik Votum der Universität Göttingen zur Durchführung der Find-AF-randomised Studie vor (Antragsnummer: 15/2/13). Damit unterliegt die Studie den Richtlinien der Good Clinical Practice (GCP) und der International Conference on Harmonisation (ICH) sowie der Deklaration von Helsinki (World Medical Association 2013).

\subsection{Datenerhebung}

Die folgende Datenerhebung wurde für alle Patienten standardisiert anhand eines für diese Studie angefertigten Case Report Form-Bogens durchgeführt (siehe Anhang).

Alle Patienten, die zum Einschluss in die Studie in die Gruppe A randomisiert wurden, erhielten direkt im Anschluss eine 10 Tage andauernde Langzeit-EKG-Untersuchung mittels zwei Kanal Holter Monitor (CardioMem CM 3000, getemed Medizin- und Informationstechnik, Teltow, Deutschland). Diese Untersuchung wurde zweimal wiederholt (drei und sechs Monaten nach Einschluss), um so die Wahrscheinlichkeit zur Diagnose eines Vorhofflimmerns weiter zu erhöhen (Wallmann et al. 2007).

Für Patienten der Kontroll-Gruppe hingegen sah das Studienprotokoll die Durchführung einer mindestens 24-Stunden andauernden EKG-Aufzeichnung vor. Die Auswahl des Monitoring war den Studienstandorten frei zur Entscheidung überlassen (z. B. LZ-EKG, Telemetrie). 
Unabhängig von der randomisierten Gruppe erhielten alle Patienten eine Blutentnahme nach Einschluss und zur ersten Follow-Up-Untersuchung nach 3 Monaten. Diese beinhaltete eine dem lokalen Studienstandort entsprechende "Standard Blutentnahme“ in der Vo Untersuchung (u. a. HbA1c, Cholesterol) sowie präspezifizierte Biomarker für kardiovaskuläre Beeinträchtigung in der V0 und V1 Untersuchung (u. a. NT-pro-BNP, NT-pro-ANP). Die Proben wurden nach Zentrifugation für weitere Untersuchungen eingefroren.

Begleitend dazu wurden anamnestisch und mit Hilfe der Patientenunterlagen allgemeine Daten zur Person wie Alter, Größe, Gewicht Geschlecht, Medikamenteneinnahme, Laborwerte (Blutbild, Glucose, Fettstoffwechsel) und bereits vorhandene Begleiterkrankungen und Risikofaktoren (insbesondere mit dem Fokus auf den Formenkreis der kardiovaskulären Erkrankungen) erhoben. Zudem wurden mit der Hilfe von standardisierten Fragebögen Daten für eine gesundheitsökonomische Beurteilung, sowie zur Lebensqualität der Patienten erfasst. Die Schwere des zu Grunde liegenden Schlaganfalls wurde mit dem National Institute of Health Stroke Scale (NIHSS), einem in der klinischen Routine gut etablierten Punktesystem, welches anhand von 11 klinisch zu überprüfenden Items (u. a. Motorik, Sensibilität) erhoben wird (Lyden 2017), objektiviert. Der aus dem Schlaganfall resultierte Grad der Behinderung wurde durch die Modified Rankin Scale (mRs) erfasst. Hier wird mittels eines Punkte Systems von 1 (keine Symptome) bis 6 (Tod) der Status des Patienten dokumentiert (van Swieten. et al. 1988).

Des Weiteren wurden bei allen Patienten die Befunde und Beurteilungen aus der klinischen Routine, wie eine Gefäßdarstellung der hirnversorgenden Gefäße (mittels duplexsonographischer Untersuchung oder alternativ röntgenologisch durch eine Computertomographie mit Kontrastmitteleinsatz), eine kraniale Bildgebung (Kernspintomographie des Kopfes oder Computertomographie des Kopfes) und ein 12-Kanal-Ruhe-EKG bei Aufnahme eingeholt und dokumentiert. Ferner wurde beim gesamten Patientenkollektiv nach Einschluss eine Echokardiographie durchgeführt, oder, wenn nicht durch das Studienpersonal erfolgt, die Daten der in der klinischen Routine in den Studienstandorten durchgeführten Echokardiographie in einen dafür konzipierten CRF-Bogen übertragen. Die Entscheidung, welche Form der Echokardiographie (TTE, TEE) beim Patienten durchgeführt wurde, oblag dem behandelnden Arzt des Patienten und wurde nicht durch das Studienpersonal getroffen. Da die Inhalte dieser Arbeit größtenteils auf den Daten dieser Untersuchung aufbauen, folgt ein Verweis auf das Kapitel 2.3, in welchem die erhobenen Daten dieser Untersuchung im Fokus stehen. 


\subsubsection{Aufnahme von Daten zur therapeutischen Konsequenz}

Klinisch relevante Ereignisse oder Änderungen der Medikation wurden zum Zeitpunkt der Entlassung, sowie bei den entsprechenden Nachfolge-Untersuchungen (V1-V3) standardisiert mittels entsprechender CRF-Bögen erfasst. Die Arztbriefe des Indexereignisses dienten im Anschluss zur Erfassung möglicher Komplikationen und therapeutischen Interventionen der erhobenen Pathologien in der Echokardiographgie. Ein Befund wurde als auffällig gewertet, wenn die durch den Untersucher in der Echokardiographie erhobene Pathologie möglichweise eine Änderung im therapeutischen Management mit sich bringen würde. Tabelle 5 gibt dabei einen Überblick, welche Befunde aus der jeweiligen Art der Echokardiographie als auffällig gewertet wurden. Die Erfassung der Parameter und Pathologien ist standartmäßig anhand eines CRFBogens bei jeder echokardiographischen Untersuchung erfolgt.

Tabelle 5: Auffällige Befunde in der Echokardiographie

\begin{tabular}{|l|l|}
\hline Transthorakale Echokardiographie & Transösophageale Echokardiographie \\
\hline Linksventrikuläre Ejektionsfraktion $<55 \%$ & Linksatrialer Thrombus (LA Thrombus) \\
Wandbewegungsstörungen/Akinesien & Vorhofohrthrombus (LAA Thrombus) \\
Linksventrikuläre Aneurysma & Klappenassoziierter Thrombus \\
Linksventrikuläre Thromben & Spontaner Echokontrast im linken Vorhof \\
Mittel-hochgradige Aortenklappenstenose & Vorhofmyxom \\
Hochgradige Mitralinsuffizienz & Endokarditis \\
Mitralklappenprolaps & Persistierendes Foramen ovale \\
& Vorhofseptumaneurysma \\
& Aortenaneurysma \\
& Aortale Plaques $>4$ mm \\
& Hochgradige Mitralinsuffizenz \\
& Mittelgradige Aortenstenose \\
& Mitralklappenprolaps \\
\hline
\end{tabular}

Weiterhin wurde aus dem Arztbrief des Indexereignisses erfasst, ob die erhobene Pathologie aus dem echokardiographischen Befund auch in der Epikrise des Patienten diskutiert wurde. Auf dieser Grundlage ließ sich bei Patienten, bei denen ein echokardiographischer Befund erhoben wurde, eine Änderung im therapeutischen Management feststellen. 


\subsection{Echokardiographische Untersuchung}

Wie bereits im Kapitel 2.2 erwähnt, wurde nach dem Studienprotokoll für alle Patienten, unabhängig von ihrer randomisierten Gruppe, eine Echokardiographie im Zuge des stationären Aufenthalts erhoben oder die entsprechenden Daten durch die klinische Routine eingeholt. Wichtig zu erwähnen ist hierbei, dass die echokardiographischen Untersuchungen größtenteils in der klinischen Routine der kardiologischen Abteilungen in den Studienzentren stattfanden. Je nach Möglichkeit und Ressourcen der verschiedenen Studienzentren wurden zusätzliche, für das Studienprotokoll als wichtig definierte Daten, ergänzt. Die Erfassung der Daten erfolgte daher durch Untersucher, welche nicht direkt im Studienprotokoll geschult waren und an unterschiedlichen Geräten. Anhand dieser Daten erfolgte die Übertragung in einen CRF-Bogen.

Die Erfassung der Daten erfolgte damit an allen Studienorten standardisiert und prospektiv. Erhobene Parameter und Messwerte können dem CRF Bogen im Anhang entnommen werden. Weitere Bestandteile der echokardiographischen Untersuchung waren die für die Fragestellung dieser Arbeit wichtige Dokumentation möglicher Emboliequellen (Akinisien, linksventrikuläre Aneurysma, linksventrikuläre Thromben), Erhebung des Klappenstatus (Klappenersatz, mögliches Vitium mit entsprechendem Schweregrad) und die Prüfung des Vorhandensein eines möglichen persistierenden Foramen ovale (Überprüfung auf einen Links-Rechts-Shunt durch agitierte Gelafundin-Lösung als Kontrastmittel und Valsalva Manöver (Lambertz und Lethen 2007)).

Wenn im Anschluss bei dem Patienten eine transösophageale Echokardiographie durchgeführt wurde, beinhaltete diese eine erweiterte Erfassung möglicher Emboliequellen (LA-Thrombus, LAAThrombus, Spontan Echokontrast im linken Vorhof, Vorhofsmyxom, Endokarditits, persistierendes Foramen ovale, Vorhofseptumaneurysma, Aortenaneurysma, Aortenplaques, Messung des maximalen Flusses im LAA) sowie die erneute Erhebung des Klappenstatus.

\subsection{Erfassung der Daten und statistische Auswertung}

Die Erfassung der Daten erfolgte primär bei allen Studienpatienten in Schriftform mittels eines angefertigten CRF-Bogens. Im Anschluss erfolgte die Übertragung der Daten (Software secuTrial ${ }^{\circledR}$ DataCapture, Version 4.4, c interActive Systems, Berlin) in eine elektronische Datenbank, welche fortan als Quelldatenbank benutzt wurde. 
Die Erfassung und Auswertung der Daten zur therapeutischen Konsequenz, wie in Kapitel 2.2.1 beschrieben, erfolgte mittels SPSS Statistics Version 23.0.0 (IBM, Chicago, Illinois, USA).

Zum Vergleich der Baseline-Charakteristika zwischen den Patientenkollektiven (Patienten mit PFO, Patienten mit LVEF < 55\%, Patienten mit Wandbewegungsstörungen) im Kapitel 3.3 wurden je nach Fragestellung und vorliegenden Messdaten der "X2-Test“, bei Auftreten einer Stichprobengröße $<5$ der „Exakte Test“ nach Fisher (jeweils bei kategorialen Variablen) und in weiteren Fällen der „Mann-Whitney-U-Test“ (bei metrischen Variablen) zum Vergleich nicht normal verteilter, unabhängiger Häufigkeiten benutzt. Ein $p$-Wert $<0,05$ wurde als ein statistisch signifikanter Unterschied der zu vergleichenden Parameter gewertet. Im Vorfeld erfolgte die Berechnung des Median sowie der Standartabweichungen der betroffenen Datenreihen.

Die graphische Aufarbeitung der Daten erfolgte mit den Programmen Microsoft Excel sowie Microsoft Powerpoint Version 2010 (Microsoft Corporation, Redmond, USA) 


\section{Ergebnisse}

\subsection{Patientenkollektiv}

Im Zeitraum von Mai 2013 bis August 2014 wurden in den vier Zentren 2848 Patienten mit den Symptomen eines kürzlich aufgetretenen Schlaganfalls nach den aufgestellten Einschluss-Kriterien

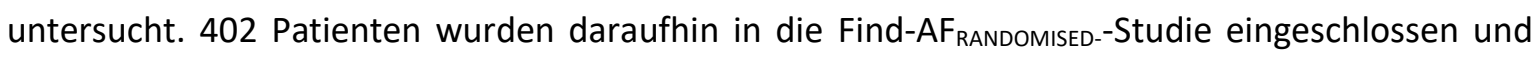
randomisiert. Vier Patienten wurden infolge eines bekannten Vorhofflimmerns und einer einseitigen hochgradigen Karotis Stenose fälschlicherweise randomisiert und nachträglich ausgeschlossen, sodass die Daten von 398 Patienten analysiert wurden.

Von diesem Patientenkollektiv ( $\mathrm{n}=398$ ) wurden 200 Patienten in die Gruppe A („enhanced and prolonged ECG-monitoring“) und 198 Patienten in die Gruppe B („usual care“) randomisiert. Die Anzahl der Echokardiographien teilte sich annähernd gleichmäßig in den Gruppen auf. So erhielten in der Gruppe A 113 (56,5\%) der Patienten eine transthorakale Echokardiographie, 29 $(14,5 \%)$ eine transösophageale Echokardiographie, 51 (25,5\%) Patienten erhielten beide Untersuchungen und sieben (3,5\%) wurden nicht untersucht. In der Gruppe B wurden 115 (58,1\%) der Patienten mittels transthorakaler Echokardiographie untersucht, $24(12,1 \%)$ erhielten eine transösophageale Echokardiographie, $51(25,7 \%)$ beide Varianten und $8(4,1 \%)$ wurden nicht untersucht. Unabhängig von der randomisierten Gruppe erhielten 330 (82,9\%) Patienten eine transthorakale Echokardiographie und 155 (38,9\%) eine transöphageale Echokardiographie. Der Hauptgrund für eine nicht durchgeführte transösophageale Echokardiographie war die fehlende Kapazität in den Studienzentren ( $\mathrm{n}=146)$.

Abbildung 2 zeigt eine Übersicht über das Patientenkollektiv und die Verteilung der Echokardiographie: 


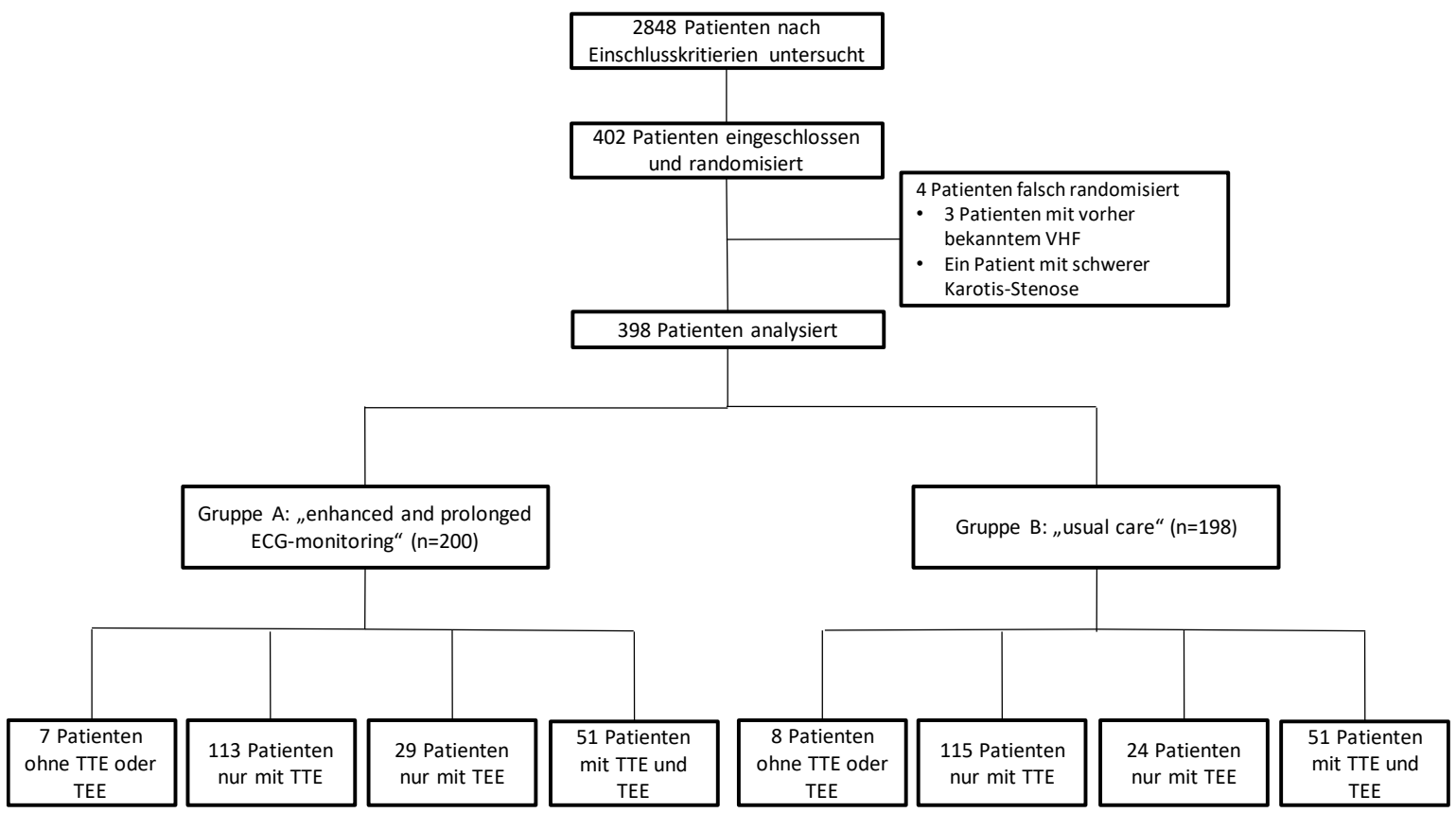

CC BY 4.0

\subsection{Gestellte Diagnosen in der Echokardiographie}

Von den mittels transthorakaler Echokardiographie untersuchten Patienten zeigten 42 (12,7\%) Patienten eine eingeschränkte linksventrikuläre Ejektionsfraktion $(<55 \%)$ und $44(13,3 \%)$ Patienten Wandbewegungsstörungen oder Akinesien als mögliche Hinweise einer zu Grunde liegenden Herzinsuffizienz, koronarer Herzkrankheit oder stattgehabte Myokardinfarkte. Zudem zeigte sich ein linksventrikuläres Aneurysma (0,3\%), acht $(2,4 \%)$ Patienten wiesen eine mindestens mittelgradige Aortenklappenstenose und vier (1,2\%) einen Mitralklappenprolaps auf.

Bei den Patienten, die eine transösophageale Echokardiographie erhielten, zeigten 33 (21,3\%) Patienten ein persistierendes Foramen ovale mit oder ohne Vorhofseptumaneurysma. Von diesen Patienten wiesen $25(16,1 \%)$ Patienten ein isoliertes persistierendes Foramen ovale und acht $(5,1 \%)$ eine Kombination aus einem persistierendem Foramen ovale und zusätzlichem Vorhofseptumaneurysma auf. Weiterhin zeigten sich bei zehn Patienten (6,5\%) aortale Plaques > $4 \mathrm{~mm}$, ein Vorhofohrthrombus (0,65\%) sowie zwei klappenassoziierte Thromben im linken (1,3\%) Ventrikel. Eine Übersicht über die Anzahl der diagnostizierten Pathologien in der Echokardiographie zeigt die Tabelle 5: 
Tabelle 6: Anzahl von Pathologien in der Echokardiographie in der Find-AF RANDOMISED $_{\text {-Studie }}$

\begin{tabular}{|c|c|c|c|}
\hline Transthorakale Echokardiographie & Anzahl & $\begin{array}{l}\text { Transösophageale } \\
\text { Echokardiographie }\end{array}$ & Anzahl \\
\hline $\begin{array}{l}\text { Linksventrikuläre Ejektionsfraktion < } \\
55 \%\end{array}$ & $42(12,7 \%)$ & $\begin{array}{l}\text { Linksatrialer Thrombus } \\
\text { (LA-Thrombus) }\end{array}$ & 0 \\
\hline Wandbewegungsstörungen/Akinesien & $44(13,3 \%)$ & $\begin{array}{l}\text { Vorhofohrthrombus } \\
\text { (LAA-Thrombus) }\end{array}$ & $1(0,7 \%)$ \\
\hline Linksventrikuläre Aneurysma & $1(0,3 \%)$ & $\begin{array}{l}\text { Spontaner Echokontrast } \\
\text { im linken Vorhof }\end{array}$ & $4(2,6 \%)$ \\
\hline Linksventrikuläre Thromben & 0 & Myxom & $1(0,7 \%)$ \\
\hline $\begin{array}{l}\text { Mindestens mittelgradige } \\
\text { Aortenklappenstenose }\end{array}$ & $8(2,4 \%)$ & Endokarditis & 0 \\
\hline Hochgradige Mitralinsuffizienz & 0 & $\begin{array}{l}\text { Persistierendes Foramen } \\
\text { ovale }\end{array}$ & $33(21,3 \%)$ \\
\hline \multirow[t]{7}{*}{ Mitralklappenprolaps } & $4(1,2 \%)$ & Vorhofseptumaneurysma & $15(9,7 \%)$ \\
\hline & & Aortenaneurysma & $4(2,6 \%)$ \\
\hline & & Aortale Plaques $>4 \mathrm{~mm}$ & $10(6,5 \%)$ \\
\hline & & $\begin{array}{l}\text { Klappenassoziierter } \\
\text { Thrombus }\end{array}$ & $2(1,3 \%)$ \\
\hline & & $\begin{array}{l}\text { Mindestens } \\
\text { mittelgradige } \\
\text { Aortenstenose }\end{array}$ & $5(3,2 \%)$ \\
\hline & & $\begin{array}{l}\text { Hochgradige } \\
\text { Mitralinsuffizenz }\end{array}$ & 0 \\
\hline & & Mitralklappenprolaps & $7(4,5 \%)$ \\
\hline
\end{tabular}

\subsection{Klinische Merkmale des Patientenkollektivs}

Die nachfolgenden Tabellen geben Aufschluss über verschiedene klinische Merkmale wie demographische Informationen über Alter und Geschlecht, Vorhandensein möglicher Vorerkrankungen, kardiovaskuläre Risikofaktoren und eingetretene Endpunkte ausgewählter

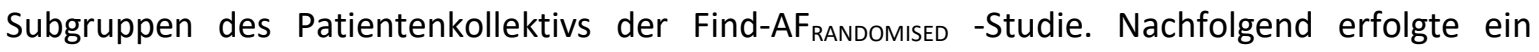


Vergleich der Basischarakteristika von Patienten mit, gegenüber ohne diagnostiziertes persistierendes Foramen ovale, sowie Patienten mit eingeschränkter Pumpfunktion (LVEF < 55\%, Wandbewegungsstörung oder Akinesie als Hinweis auf eine zugrundeliegende konorare Herzkrankheit) gegenüber Patienten mit normwertiger Pumpfunktion.

Tabelle 7: Klinische Eigenschaften des Find-AF Median oder der absoluten Anzahl (mit prozentualem Anteil)

\begin{tabular}{|c|c|c|}
\hline & & Alle Patienten $n=398$ \\
\hline \multicolumn{2}{|l|}{ Durchschnittliches Alter } & $72,7 \pm 7,5$ \\
\hline \multicolumn{2}{|l|}{ Weibliches Geschlecht } & $160(40,1 \%)$ \\
\hline \multicolumn{2}{|l|}{ NIHSS (IQR) } & $\begin{array}{l}3(1 ; 5) \\
n=397\end{array}$ \\
\hline \multicolumn{2}{|l|}{ MRS-Score } & $\begin{array}{l}2(1 ; 3) \\
n=398\end{array}$ \\
\hline \multirow[t]{4}{*}{ CVR-Faktoren } & Arterielle Hypertension & $316(79,2 \%)$ \\
\hline & Diabetes Mellitus & $108(27,1 \%)$ \\
\hline & Nikotinabusus & $70(17,6 \%)$ \\
\hline & Hyperlipoproteinämie & $164(41,1 \%)$ \\
\hline \multirow[t]{6}{*}{ Vorerkrankungen } & Schlaganfall & $77(19,3 \%)$ \\
\hline & TIA & $31(7,8 \%)$ \\
\hline & Bekannte KHK & $61(15,3 \%)$ \\
\hline & Myokardinfarkt & $38(9,5 \%)$ \\
\hline & Herzinsuffizienz & $20(5 \%)$ \\
\hline & pAVK & $23(5,8 \%)$ \\
\hline \multirow[t]{5}{*}{ Endpunkte } & Kardiovaskulärer Tod & $5(1,3 \%)$ \\
\hline & $\begin{array}{l}\text { Sonstige } \\
\text { Todesursache }\end{array}$ & $14(3,5 \%)$ \\
\hline & Ischämischer Schlaganfall & $21(5,3 \%)$ \\
\hline & TIA & $16(4 \%)$ \\
\hline & Myokardinfarkt & $6(1,5 \%)$ \\
\hline \multirow[t]{5}{*}{ TOAST-Klassifikation } & Zerebrale Makroangiopathie & $7(1,8 \%)$ \\
\hline & Kardioembolisch & $75(18,8 \%)$ \\
\hline & Zerebrale Mikroangiopathie & $118(29,6 \%)$ \\
\hline & Spezielle Ätiologie & $1(0,3 \%)$ \\
\hline & Unklare Ätiologie & $197(49,4 \%)$ \\
\hline
\end{tabular}

Die Patienten der Find-AF $F_{\text {RANDOMISED }}$-Studie befanden sich mehrheitlich in der siebten Lebensdekade $(72,7$ SD $\pm 7,5)$ und zeigten mit einem NIHSS Score von drei (IQR 1;5) im Median und 3,66 (SD $\pm 4,0)$ im Durchschnitt überwiegend die Schlaganfall-Schwere eines Minor Strokes (Punktzahl 1-4). 77 (19,3\%) Patienten erlitten bereits im Vorfeld einen Schlaganfall. Bei 61 (15,3\%) Patienten bestand bereits eine koronare Herzkrankheit. Eine große Anzahl der Patienten wies mit 316 (79,4\%) Fällen von arterieller Hypertonie und 108 (27,1\%) Diabetikern ein kardiovaskuläres Risikoprofil auf. 21 (5,3\%) erlitten im Laufe der Studie einen weiteren Schlaganfall. Ätiologisch (Einteilung nach der TOAST-Klassifikation) zeigten sich mit $197(49,4 \%)$ mehrheitlich Hirninfarkte 
unklarer Ätiologie sowie 118 (29,6\%) mikroangiopathischer Genese. Sieben (1,8\%) Patienten erlitten einen Hirninfarkt makroangiopathischer Genese, bei 75 (18,8\%) Patienten konnte eine kardioembolische Ursache diagnostiziert werden.

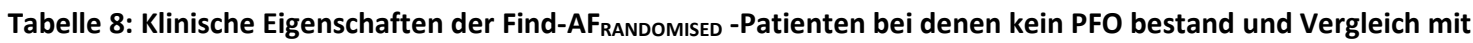
Patienten, bei denen ein PFO diagnostiziert wurde. Angabe des Durchschnitts (Alter), der Anzahl (mit prozentualem Anteil) und des Median, $p<0,05$ wurde als signifikant gewertet.

\begin{tabular}{|c|c|c|c|c|}
\hline & & $\begin{array}{l}\text { Kein PFO } n= \\
365\end{array}$ & $\begin{array}{l}\text { PFO } \\
n=33\end{array}$ & $\begin{array}{l}\text { p-Wert } \\
\text { Kein PFO vs. } \\
\text { PFO }\end{array}$ \\
\hline \multicolumn{2}{|c|}{ Durchschnittliches Alter } & $73 \pm 7,375$ & $70 \pm 7,5$ & 0,001 \\
\hline \multicolumn{2}{|c|}{ Weibliches Geschlecht } & $146(40 \%)$ & $14(42,4 \%)$ & 0,786 \\
\hline \multicolumn{2}{|c|}{ NIHSS (IQR) } & $\begin{array}{l}3(1 ; 5) \\
n=364\end{array}$ & $3(2 ; 5,5)$ & 0,106 \\
\hline \multicolumn{2}{|l|}{ MRS-Score } & $\begin{array}{l}2(1 ; 3) \\
n=365\end{array}$ & $2(1 ; 3)$ & 0,528 \\
\hline \multirow[t]{4}{*}{ CVR-Faktoren } & Arterielle Hypertension & $291(79,7 \%)$ & $25(75,8 \%)$ & 0,589 \\
\hline & Diabetes Mellitus & $103(28,2 \%)$ & $5(15,2 \%)$ & 0,106 \\
\hline & Nikotinabusus & $61(16,7 \%)$ & $9(27,3 \%)$ & 0,192 \\
\hline & Hyperlipoproteinämie & $155(42,5 \%)$ & $9(27,3 \%)$ & 0,089 \\
\hline \multirow[t]{6}{*}{ Vorerkrankungen } & Schlaganfall & $71(19,5 \%)$ & $6(18,2 \%)$ & 0,860 \\
\hline & TIA & $28(7,7 \%)$ & $3(9,1 \%)$ & 0,733 \\
\hline & Bekannte KHK & $56(15,3 \%)$ & $5(15,2 \%)$ & 0,977 \\
\hline & Myokardinfarkt & $35(9,5 \%)$ & $3(9,1 \%)$ & 1,000 \\
\hline & Herzinsuffizienz & $20(5,5 \%)$ & 0 & 0,394 \\
\hline & pAVK & $23(6,3 \%)$ & 0 & 0,241 \\
\hline \multirow[t]{5}{*}{ Endpunkte } & Kardiovaskulärer Tod & $5(1,4 \%)$ & 0 & 1,000 \\
\hline & $\begin{array}{l}\text { Sonstige } \\
\text { Todesursache }\end{array}$ & $14(3,8 \%)$ & 0 & 0,617 \\
\hline & Ischämischer Schlaganfall & $21(5,8 \%)$ & 0 & 0,240 \\
\hline & TIA & $14(3,8 \%)$ & $2(6,1 \%)$ & 0,633 \\
\hline & Myokardinfarkt & $6(1,6 \%)$ & 0 & 1,000 \\
\hline
\end{tabular}

Im Vergleich mit den Patienten, bei denen ein persistierendes Foramen ovale diagnostiziert wurde, zeigen sich bis auf das Alter (Median 70 SD $\pm 7,5$ ) keine wesentlichen Unterschiede zwischen den Patientengruppen. 


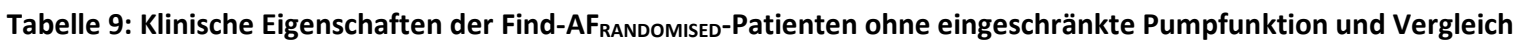
mit Patienten, bei denen eine eingeschränkte Pumpfunktion diagnostiziert wurde. Angabe des Durchschnitts (Alter), der Anzahl (mit prozentualem Anteil) und des Median, $p<0,05$ wurde als signifikant gewertet.

\begin{tabular}{|c|c|c|c|c|}
\hline & & $\begin{array}{l}\text { Keine } \\
\text { eingeschränkte } \\
\text { Pumpfunktion* } \\
n=\mathbf{3 4 0}\end{array}$ & $\begin{array}{l}\text { Eingeschränkte } \\
\text { Pumpfunktion* } \\
\text { n = 58 }\end{array}$ & $\begin{array}{l}\text { p-Wert } \\
\text { Eingeschränkte } \\
\text { Pumpfunktion* } \\
\text { vs. keine } \\
\text { eingeschränkte } \\
\text { Pumpfunktion* }\end{array}$ \\
\hline \multicolumn{2}{|c|}{ Durchschnittliches Alter } & $\begin{array}{l}72 \pm 7,3 \\
(n=339)\end{array}$ & $74 \pm 8,3$ & 0,093 \\
\hline \multicolumn{2}{|c|}{ Weibliches Geschlecht } & $145(42,6 \%)$ & $15(25,9 \%)$ & 0,016 \\
\hline \multicolumn{2}{|l|}{ NIHSS (IQR) } & $2(1 ; 5)$ & $3(2 ; 7)$ & 0,005 \\
\hline \multicolumn{2}{|l|}{ MRS-Score } & $2(1 ; 3)$ & $2(1 ; 3)$ & 0,259 \\
\hline \multirow[t]{4}{*}{ CVR-Faktoren } & $\begin{array}{l}\text { Arterielle } \\
\text { Hypertension }\end{array}$ & $268(78,8 \%)$ & $48(84,2 \%)$ & 0,493 \\
\hline & Diabetes Mellitus & $83(24,4 \%)$ & $25(43,1 \%)$ & 0,003 \\
\hline & Nikotinabusus & $152(44,7 \%)$ & $34(58,6 \%)$ & 0,05 \\
\hline & Hyperlipoproteinämie & $133(39,1 \%)$ & $31(53,4 \%)$ & 0,04 \\
\hline \multirow[t]{6}{*}{ Vorerkrankungen } & Schlaganfall & $63(18,5 \%)$ & $14(24,1 \%)$ & 0,318 \\
\hline & TIA & $27(7,9 \%)$ & $4(6,9 \%)$ & 1,00 \\
\hline & Bekannte KHK & $42(12,4 \%)$ & $19(32,8 \%)$ & 0,000 \\
\hline & Myokardinfarkt & $25(7,0 \%)$ & $19(32,8 \%)$ & 0,000 \\
\hline & Herzinsuffizienz & $13(3,7 \%)$ & $9(15,5 \%)$ & 0,000 \\
\hline & pAVK & $17(5,0 \%)$ & $6(10,3 \%)$ & 0,107 \\
\hline \multirow[t]{6}{*}{ Endpunkte } & Kardiovaskulärer Tod & $2(0,6 \%)$ & $3(5,2 \%)$ & 0,024 \\
\hline & $\begin{array}{l}\text { Sonstige } \\
\text { Todesursache }\end{array}$ & $9(2,6 \%)$ & $5(8,6 \%)$ & 0,039 \\
\hline & $\begin{array}{l}\text { Ischämischer } \\
\text { Schlaganfall }\end{array}$ & $18(5,3 \%)$ & $3(5,2 \%)$ & 1,00 \\
\hline & TIA & $15(4,4 \%)$ & $1(1,7 \%)$ & 0,486 \\
\hline & Myokardinfarkt & $5(1,5 \%)$ & $1(1,6 \%)$ & 1,00 \\
\hline & $\begin{array}{l}\text { Vorhofflimmern nach } \\
12 \text { Monaten }\end{array}$ & $34(10 \%)$ & $6(10,3 \%)$ & 0,936 \\
\hline
\end{tabular}

*: Eingeschränkte Pumpfunktion: LVEF < 55\% (TTE) sowie regionale Wandbewegungsstörungen/Akinesien (TTE)

Im Vergleich mit den Patienten, welche keine Einschränkung in der Pumpfunktion aufwiesen, zeigte sich, dass unter den Patienten, bei denen eine eingeschränkte Pumpfunktion diagnostiziert wurde, ein geringerer Anteil des weiblichen Geschlechts (15; 25,9\%), sowie eine erhöhte Schlaganfall-Schwere (NIHSS Punkte Median 3 IQR 2;7, Mittelwert 5,02) vorhanden war. Des Weiteren zeigte sich, dass bei einer erhöhten Anzahl der Patienten dieser Gruppe bereits kardiovaskuläre Vorerkrankungen bekannt waren. So wiesen 19 (32,8\%) Patienten eine koronare Herzkrankheit auf, 19 (32,8\%) erlitten bereits einen Myokardinfarkt und bei neun (15,5\%) ist eine 
Herzinsuffizienz bereits bekannt gewesen. Auch die Inzidenz kardiovaskulärer Risikofaktoren zeigte sich bei $25(43,1 \%)$ Patienten mit einer Diabetes sowie 31 (53,4\%) Patienten mit einer Hyperlipoproteinämie erhöht. Es zeigte sich weiterhin, dass Patienten aus dieser Gruppe im Laufe der Studie eine erhöhte Sterblichkeit aufwiesen (kardiovaskulärer Tod: drei Patienten (5,2\%), sonstige Todesursache: fünf Patienten (8,6\%).

\subsection{Therapeutische Konsequenzen durch die Echokardiographie}

Insgesamt wurden im Zuge der Find-AF $F_{\text {RANDOMISED }}$-Studie 495 echokardiographische Untersuchungen durchgeführt (155 TEE und 330 TTE). Dabei enthielten 110 Befunde dieser Untersuchungen (53 TEE und 57 TTE) mindestens eine Diagnose, welche im Kapitel 2.4 vorher als auffällig festgelegt wurde. Zu einer therapeutischen Änderung kam es insgesamt bei 23 Patienten (4 TTE und 19 TEE). Für 472 Patienten hatte die echokardiographische Untersuchung keine Auswirkung auf die bestehende therapeutische Betreuung. Eine Übersicht über die Art und Anzahl der therapeutischen Änderung gibt Abbildung 3:

Abbildung 3: Art und Anzahl der therapeutischen Änderungen durch die Echokardiographie in der Find-AF Studie.

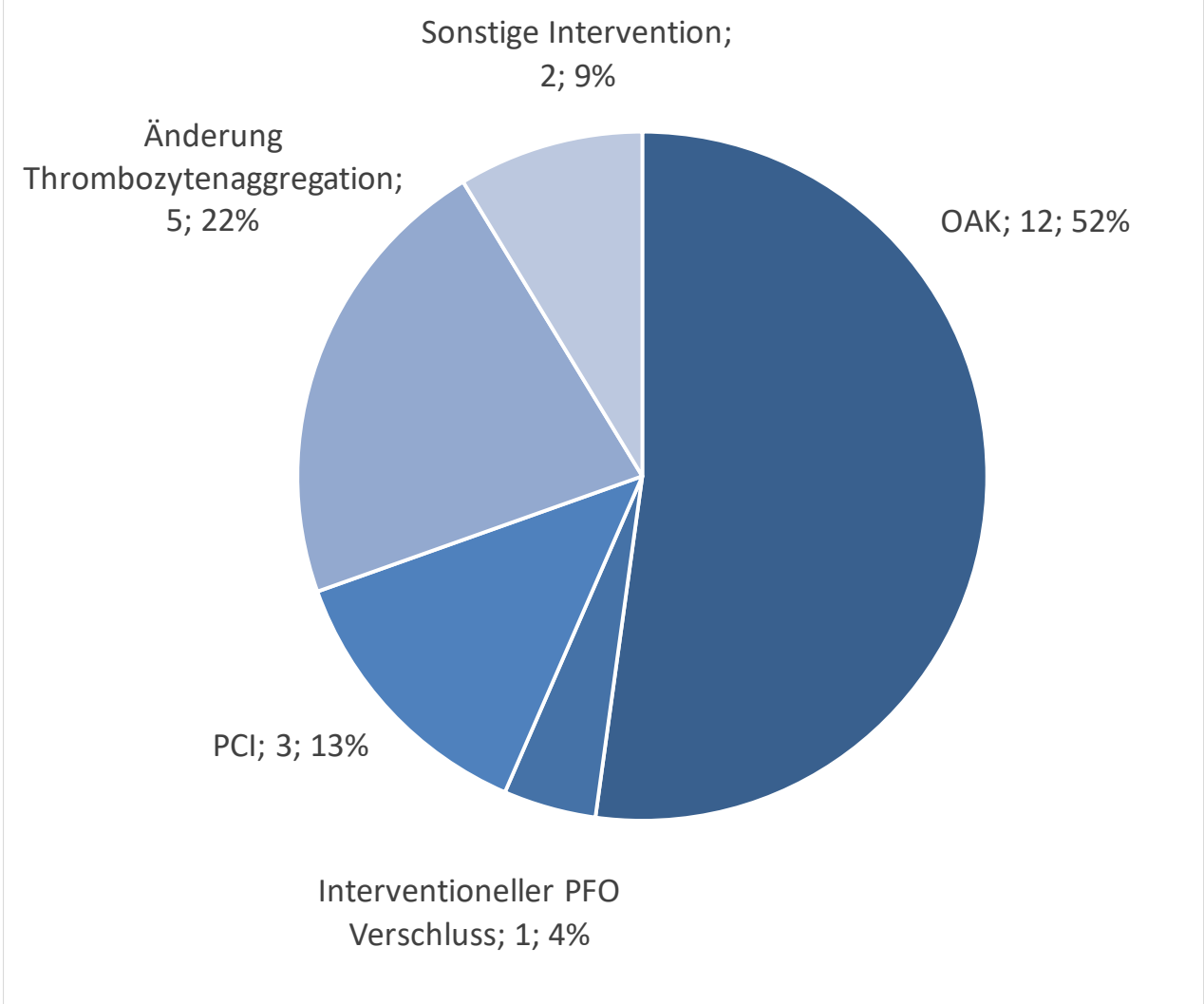


Bei drei (13\%) Patienten wurde die Indikation zur perkutanen koronaren Intervention gestellt. Ein Patient wurde auf Grund einer unklaren Struktur an der Mitralklappe zur dringlichen operativen Versorgung in die Thorax-Chirurgie verlegt. Ein weiterer wurde auf Grund einer schweren Aortenklappenstenose operativ versorgt (zwei sonstige Interventionen 9\%). Fünf (22\%) Patienten erhielten eine Änderung ihrer medikamentösen Thrombozytenaggregation. Bei einem (4\%) Patienten wurde ein persistierendes Foramen ovale interventionell verschlossen.

Am häufigsten resultierte der Beginn einer oralen Antikoagulation (12; 52\%). Gründe hierfür waren zum einen durch die Echokardiographie diagnostizierte Thromben. Ein Patient zeigte ein Thrombus im Vorhofohr (LAA Thrombus), ein weiterer wies einen ulzerierten aortalen Plaque mit Thrombus-Auflagerung auf. Bei einem Patienten konnte thrombotisches Material auf der Aortenklappe nachgewiesen werden.

Mehrheitlich $(9 ; 39 \%)$ erfolgte die Indikationsstellung einer oralen Antikoagulation infolge der Diagnose eines persistierenden Foramen ovale und/oder Vorhofseptumaneurysma. Bei zwei Patienten mit einem Vorhofseptumaneurysma wurde eine orale Antikoagulation nachträglich eingeleitet, da ein relevanter Rechts-Links-Shunt nachgewiesen wurde, ohne dass in der transösophagealen Echokardiographie ein persistierendes Foramen ovale dargestellt oder im Befund erwähnt wurde.

Die Rate an Indikationsstellung zur oralen Antikoagulation differierte dabei abhängig vom Studienzentrum (35.7\%, 5.6\%, 50.0\%, 0.0\% für Studienzentrum 1-4). Weiterhin ist zu erwähnen, dass je nach Studienzentrum ein eigenes therapeutisches Konzept bei einem neu diagnostizierten persistierenden Foramen ovale verfolgt wurde. Eine Übersicht über die Anzahl und Art der Therapie des persistierenden Foramen ovale in den verschiedenen Studienzentren zeigt Tabelle 10: 
Tabelle 10: Vergleich der therapeutischen Maßnahmen bei Diagnose eines persistierenden Foramen ovale in den Studienzentren.

\begin{tabular}{|c|c|c|c|c|}
\hline Studienzentrum & $\begin{array}{l}\text { Anzahl } \\
\text { PFO }\end{array}$ & $\begin{array}{l}\text { Orale } \\
\text { Antikoagulation }\end{array}$ & $\begin{array}{l}\text { Änderung der } \\
\text { Thrombozytenaggregation }\end{array}$ & $\begin{array}{l}\text { Interventioneller } \\
\text { PFO Verschluss }\end{array}$ \\
\hline $\begin{array}{l}\text { Universitätsmedizin } \\
\text { Göttingen }\end{array}$ & 8 & 4 & 0 & 0 \\
\hline $\begin{array}{l}\text { Universitätsmedizin } \\
\text { Mainz }\end{array}$ & 18 & 1 & $3 *$ & 0 \\
\hline $\begin{array}{l}\text { Nordwest- } \\
\text { Krankenhaus } \\
\text { Sanderbusch }\end{array}$ & 2 & 1 & 0 & 0 \\
\hline $\begin{array}{l}\text { Horst Schmidt } \\
\text { Kliniken Wiesbaden }\end{array}$ & 5 & 1 & $2^{* *}$ & 1 \\
\hline
\end{tabular}

Ein diagnostizierter spontaner Echokontrast fand keine Beachtung im Sinne einer Dokumentation im neurologischen Arztbrief noch einer Intervention. Gleiches galt für Fälle diagnostizierter Mitralprolapse. Abbildung 4 zeigt die Verteilung von gestellten Pathologien und die im Verhältnis als Konsequenz durchgeführten Interventionen der Studienpatienten.

Abbildung 4: Anzahl der gestellten Diagnosen in der Echokardiographie und eingeleiteter therapeutischen Konsequenzen im Vergleich

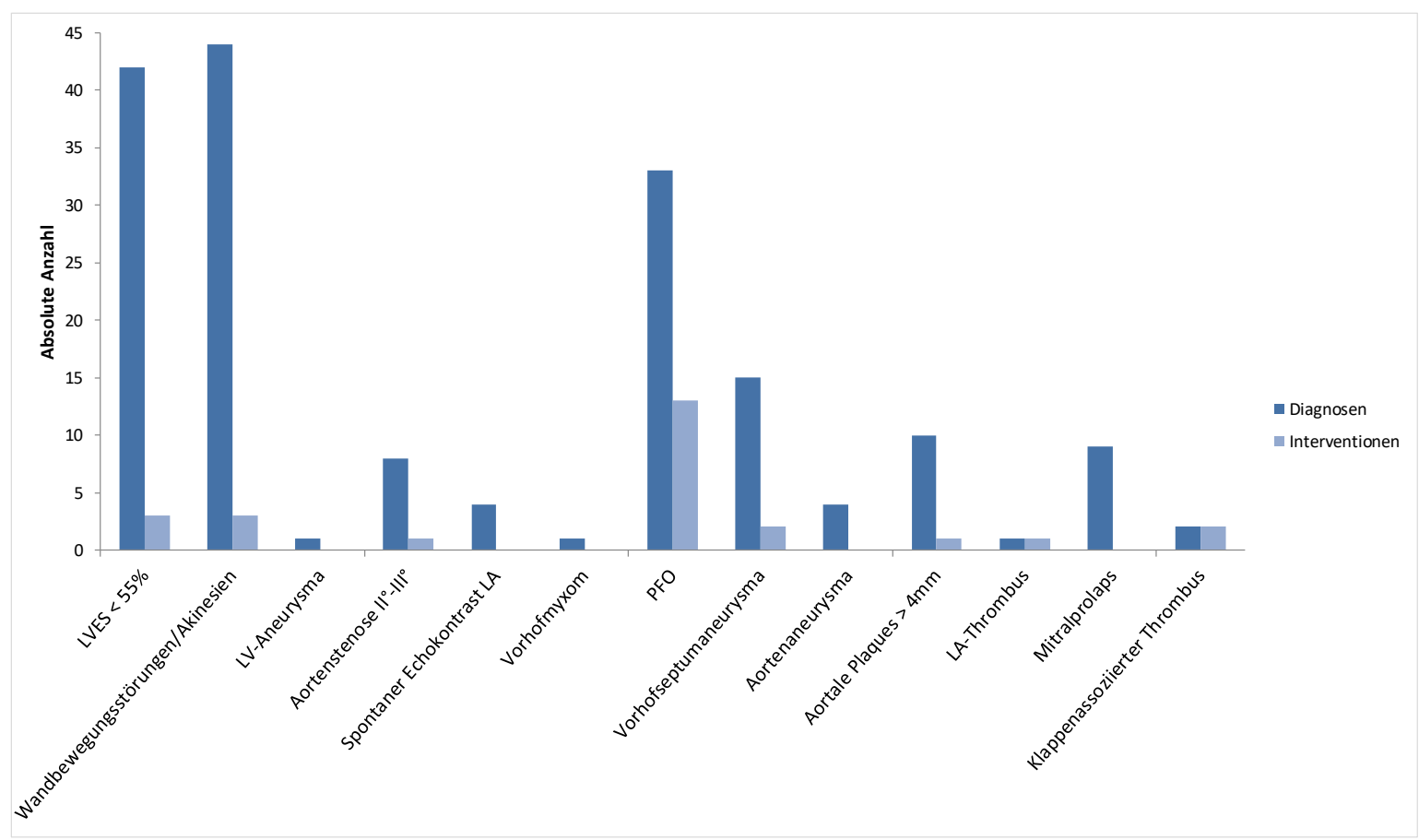




\subsection{Effektivität der Echokardiographie}

Zusammenfassend wurden 181 Diagnosen bei 112 Patienten durch die Echokardiographie gestellt, 99 davon durch eine transthorakale, 82 durch eine thransösophageale Untersuchung.

Gemessen an der in Kapitel 1.1.3 gezeigten Einteilung, konnten durch die transthorakale Echokardiographie null und durch die transösophageale Echokardiographie vier (4,9\%; 4/82) Hoch-Risiko-Pathologien, bezogen auf das Embolie-Risiko, nachgewiesen werden. In der transthorakalen Echokardiographie zeigten sich 48 (48,5\%; 48/99) und in der transösophagealen Echokardiographie 59 (67,1\%; 55/82) Pathologien mit einem niedrigen oder unklaren Risiko für Embolien.

Insgesamt kam es in 23 von 485 Fällen (4,74\%, transthorakale Echokardiographie und transösophageale Echokardiogpraphie) zu einer Änderung im therapeutischen Management: Vier entfielen auf die transthorakale (Rate an Interventionen: 1,21\% (4/330)) und 19 auf die thransösophageale (Rate an Interventionen: 12,26\% (19/155)) Echokardiographie.

\subsubsection{Vergleich der Anzahl therapeutischer Konsequenzen zwischen der Echokardiographie und dem Langzeit-EKG}

Vergleicht man die beiden untersuchten Methoden der Echokardiographie (TTE und TEE) mit den Langzeit-EKG-Methoden dieser Studie (10 Tage Langzeit-EKG der Gruppe A vs. Routine 24Stunden-EKG der Gruppe B), gemessen an der Anzahl der therapeutischen Konsequenzen, erhält man folgende Ergebnisse:

Im Zuge der Find-AF RANDomised -Studie erhielten 199 von 200 Patienten aus der Interventionsgruppe ein Langzeit-EKG für zehn Tage. Innerhalb der Gruppe konnte bei 27 Patienten (13,5\%; 27/199) nach Einschluss in die Studie die Diagnose eines Vorhofflimmerns gestellt werden. In der Kontroll-Gruppe gelang dies durch mindestens 24 stündige EKG Ableitung bei 12 Patienten (6,1\%; 12/198). Alle Patienten, bei denen ein Vorhofflimmern detektiert wurde, erhielten als Konsequenz eine orale Antikoagulation. Abbildung 5 zeigt den Vergleich der verschiedenen diagnostischen Methoden anhand der Anzahl an therapeutischen Konsequenzen. 
Abbildung 5: Vergleich der diagnostischen Methoden anhand der Anzahl therapeutischer Konsequenzen, modifiziert nach Wasser et al. 2019.

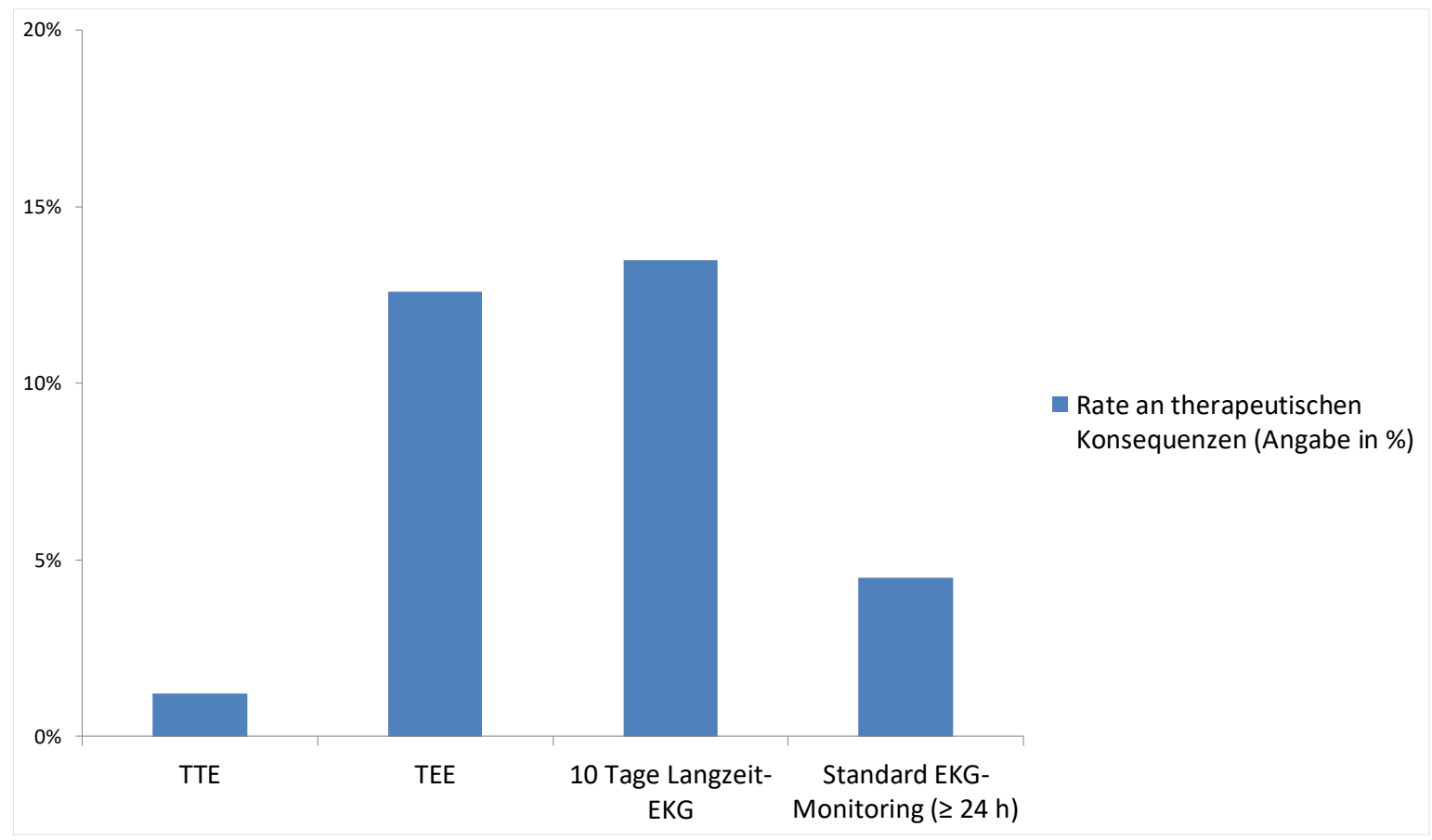

CC BY 4.0 


\section{Diskussion}

Ziel dieser Arbeit war es, den Stellenwert der Echokardiographie (transthorakal und transösophageal) in der Diagnostik des ischämischen Schlaganfalls anhand direkter Konsequenzen für den Patienten (z. B. weiterführende Diagnostik, Änderung des bestehenden therapeutischen Regimes) zu bewerten. Hierzu wurden die echokardiographischen Daten verwendet, welche im Rahmen der multizentrischen, randomisierten und kontrollierten Find-AF RANDOMISED -Studie erhoben wurden.

\subsection{Studienergebnisse im wissenschaftlichen Vergleich}

Neben dieser Arbeit beschäftigten sich zahlreiche Studien mit der Fragestellung nach der Wertigkeit der Echokardiographie in der Diagnostik des ischämischen Schlaganfalls. Insbesondere die Rolle der transösophagealen Echokardiographie gelangt auf Grund der Diskrepanz zwischen einem hohen zeitlichen und finanziellen Aufwand und den häufig unklaren resultierenden Konsequenzen (z. B. auf Grund mangelhafter Datenlage zur Behandlung der diagnostizierten Embolie-Quelle) in den Fokus der Studien (Harloff et al. 2006; Warner 1996; Katsanos et al. 2016; Rauh et al. 1996; Strandberg et al. 2002).

So untersuchten Harloff et al. (2006) Möglichkeiten zum optimalen Einsatz der transösophagealen Echokardiographie und resultierende therapeutische Konsequenzen aus den Befunden von 503 Patienten, die einen ischämischen Schlaganfall erlitten haben. Nach Ausschluss der Patienten, bei denen die Schlaganfall-Ätiologie anhand der TOAST-Klassifikation festgestellt werden konnte und keine Kontraindikation zur oralen Antikoagulation bestand, erhielten die verbliebenen 212 Patienten eine transösophageale Echokardiographie. Als Konsequenz wurden im Anschluss 65 (30,6\%; 65/212) der Patienten dieses Patientenkollektivs („stroke of undetermined etiology“) auf Grund der Befunde der transösophagealen Echokardiographie Untersuchung oral antikoaguliert. Diese im Vergleich zu den Ergebnissen der vorliegenden Arbeit hohe Anzahl $(12,26 \% ; 23 / 155)$ resultiert aus einer sehr breiten, nicht leitlinienbegründeten Auslegung der Indikationsstellung zur oralen Antikoagulation bei Pathologien, in denen die optimale therapeutische Strategie noch Gegenstand der Forschung ist. So wurden konsequent Patienten mit spontanem Echokontrast ( $\mathrm{n}=$ $5)$, verlangsamtem Blutfluss im Vorhofohr $(n=1)$, PFO $(n=25)$, Vorhofseptumaneurysma $(n=2)$ und ein persistierendes Foramen ovale in Kombination mit einem Vorhofseptumaneurysma ( $\mathrm{n}=$ 21) einer oralen Antikoagulation unterzogen (Harloff et al. 2006). 
Strandberg et al. (2002) zeigten mit ihren Daten, dass $8 \%$ ihrer Patienten ( $n=457$ ) im Sinusrhythmus und ohne kardiologische Vorerkrankungen auf der Basis einer transösophagealen Echokardiographie eine orale Antikoagulation erhielten und empfehlen daher in dieser Gruppe einen breiten Einsatz dieser Untersuchung. Genaue Angaben, welche einzelne Pathologien in diesen Fällen zur Entscheidung der Antikoagulation geführt haben, fehlen jedoch.

Katsanos et al. (2016) kamen in ihrer Studie zur Untersuchung der Wertigkeit einer transösopghagealen Echokardiographie bei Schlaganfall Patienten mit der Ätiologie eines „Embolic stroke of undetermined source" (ESUS) (Hart et al. 2014) zu dem Ergebnis, dass 16\% (10/61) der untersuchten Patienten im Sinne einer therapeutischen Konsequenz profitierten. Zu diesen Änderungen zählten neben einer oralen Antikoagulation $(n=5)$ auch die Einleitung einer antibiotischen Therapie infolge einer Endokarditis $(n=2)$ oder interventionellen Verschlüssen eines persistierenden Foramen ovale $(n=3)$. In einer zusätzlich durchgeführten Meta-Analyse von 3562 Patienten mit einem akuten ischämischen Schlaganfall zeigte sich, dass eine Indikation zur oralen Antikoagulation bei 8,7\% der Patienten infolge einer transösophagealen Echokardiographie resultierte. Interessanterweise sank dieser Anteil auf 4,2\%, sobald die Indikationsstellung zur oralen Antikoagulation leitliniengerecht durchgeführt wurde. Die Autoren benennen daher selbstkritisch als wichtige Limitation ihrer Meta-Analyse, dass in den ausgewerteten Studien keine konsistente Strategie z. B. zur Indikationsstellung zur oralen Antikoagulation zu erkennen war. Viele dieser berichteten therapeutischen Endscheidungen seien nicht leitliniengerecht durchgeführt worden (Katsanos et al. 2016).

Die Untersuchung der Wirksamkeit der transösophagealen Echokardiographie in der Subgruppe der kryptogenen Schlaganfälle (Saver 2016) erbrachte ebenfalls heterogene Ergebnisse. So kamen McGrath et al. (2014) in ihrer Metaanalyse zu dem Schluss, dass keine klare Empfehlung zur Durchführung einer transösophagealen Echokardiographie bei Patienten mit einem kryptogenen Schlaganfall gegeben werden kann. Zwar ließen sich viele Befunde erheben und diese führten auch häufig zu therapeutischen Konsequenzen wie z. B. einer oralen Antikoagulation, jedoch fielen diese Entscheidungen nicht auf Grundlage etablierter Leitlinien (McGrath et al. 2014). Auf der anderen Seite argumentierten Knebel et al. (2009) für eine hohe Effektivität der transösophagealen Echokardiographie bei kryptogenen Schlaganfällen aufgrund der hohen Anzahl an Befunden, die einen ätiologischen Zusammenhang zum erlittenen Schlaganfall zulassen und somit spezifische therapeutische Konsequenzen nach sich ziehen. Auf welcher Grundlage die Wahl der therapeutischen Konsequenzen basiert, erwähnen die Autoren der Studie nicht.

Im Gegensatz zu den anderen hier vorgestellten Studien schlussfolgerte Warner (1996) in einer Untersuchung von 106 Schlaganfall Patienten durch eine transösophageale Echokardiographie, 
dass ein breiter Einsatz dieser Untersuchung möglicherweise nicht kosteneffektiv sei, und daher kritisch hinterfragt werden sollte (Warner 1996). Die Studie diagnostizierte bei $35 \%$ der untersuchten Patienten eine mögliche Emboliequelle durch die transösophageale Echokardiographie. Ein Großteil dieser Befunde wurde jedoch bei Patienten mit einem bekannten Vorhofflimmern diagnostiziert, bei denen ohnehin die Indikation zur oralen Antikoagulation bestand. Bei den verbleibenden Patienten, die sich im Sinusrhythmus befanden, hat die Untersuchung keine Konsequenz, da es für die in der Echokardiographie erhobenen Befunde bisher keine empfohlene Therapie bekannt ist (Warner 1996).

Die Diversität der Ergebnisse aus den vorliegenden Studien zeigt die Uneinigkeit in der optimalen Behandlung möglicher kardialer Emboliequellen. Indikationen zur oralen Antikoagulation wurden häufig unabhängig von wissenschaftlicher Evidenz gestellt. Dies liegt natürlich auch an der mangelhaften Datenlage zum Zeitpunkt der Erstellung der Studien zur Behandlung diagnostizierter Emboliequellen, die bis heute besteht. Beispielsweise wurde eine Studie zur optimalen Therapie von Aortalen Plaques $>4 \mathrm{~mm}$ aufgrund unzureichender Rekrutierung vorzeitig abgebrochen (Amarenco et al. 2014). Seit kurzer Zeit liegen ausreichend Erkenntnisse zur Behandlung eines persistierenden Foramen ovale bei Patienten mit einem kryptogenen Schlaganfall und einem Alter $<60$ Jahre vor (Diener et al. 2018). Anhand der bestehenden Datenlage lässt sich derzeit kein Vorteil einer oralen Antikoagulation gegenüber einer Thrombozytenaggregationshemmung ableiten (Diener et al. 2018), wie sie in einigen Studien Anwendung findet (Harloff et al. 2006).

Des Weiteren wird bei der Mehrheit dieser Studien der Wert der Echokardiographie, weitestgehend transösophageal, allein anhand der Anzahl an Indikationsstellungen zur oralen Antikoagulation gemessen. In der vorliegenden Arbeit mit den Daten der Find-AF $F_{\text {RANDOMISED }}$-Studie wurden auch andere therapeutische Ansätze wie Koronarangiographien oder Änderungen an der Thrombozytenaggregation berücksichtigt. Im Vergleich der Studien fällt auch auf, dass die Wirksamkeit der Echokardiographie häufig an verschiedenen Untergruppen (ESUS, Stroke of undetermined etiology, Sinusrhythmus ohne kardiologische Vorerkrankungen, kryptogener Schlaganfall) untersucht wurde. Es zeigen sich auch große Unterschiede in der Zusammensetzung und damit in den Charakteristika der Patientenkollektive wie beispielsweise im Durchschnittsalter (Harloff et al. (2006): 58,2 $\pm 13,9$; Katsanos et al. (2016): 44,3 $\pm 11,5$ ). Eine Vergleichbarkeit der Studien wird dadurch erheblich erschwert. 


\subsection{Bewertung des Patientenkollektivs und der erstellten Patientengruppen}

Die Ergebnisse der Find-AF $F_{\text {RANDOMISED }}$-Studie sind mit dem Hintergrund der Einschlusskriterien zu bewerten (siehe Kapitel 2.1). Diese hatten dadurch erheblichen Einfluss auf das Patientenkollektiv (Alter: $72,7 \pm 7,5$; Patienten im Sinusrhythmus, keine Patienten mit einer Indikation oder Kontraindikation für eine orale Antikoagulation). Weiterhin wurde in der vorliegenden Arbeit auch Wert auf die Befunde und Auswirkungen der transthorakalen Echokardiographie gelegt. Mit 330 durchgeführten transthorakalen und 155 transösophagealen Untersuchungen wurden hier weniger Patienten untersucht als bei einem Großteil der anderen Studien. Auch wurden die Daten im Gegensatz zu einigen anderen Studien prospektiv erhoben.

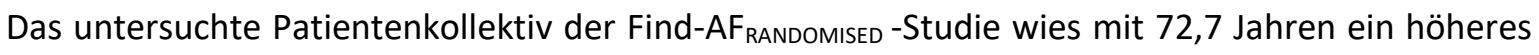
Durchschnittsalter als die meisten vergleichbaren Studien zur Echokardiographie auf. Zusätzlich dazu zeigen ein Überwiegen des männlichen Geschlechts (40,1\% weiblich) und ein ausgeprägtes kardiovaskuläres Risikoprofil (79,2\% arterieller Hypertonus; 41,1\% Hypercholesterinämie) deutlich, dass Patienten, die einen Schlaganfall erlitten haben, mit einem hohen Risiko behaftet sind an weiteren kardiovaskulären Erkrankungen anderer Organsysteme (z. B. koronare Herzkrankheit, periphere arterielle Verschlusskrankheit) zu leiden oder sie zukünftig zu erwerben, da diese sich die gleichen Risikofaktoren teilen (Bhatt et al. 2006; Lusis 2000). Mit diesem Hintergrund unterstreichen die Ergebnisse dieser Studie explizit, dass ein vaskulär- ischämisches Ereignis wie eine transitorisch ischämische Attacke oder ein Schlaganfall als ein Index Ereignis für jeden Patienten gewertet werden sollte, weitere kardiovaskuläre Diagnostik einzuleiten, um eine Bestandsaufnahme zu erstellen und den Patienten als kardiovaskulären Risikopatienten zu identifizieren (Laufs et al. 2010). Dadurch könnten zum einen entsprechende sekundärprophylaktische Maßnahmen wie eine Optimierung des Risikoprofils (Nikotinkarenz, moderate Bewegung, Ernährung), oder eine medikamentöse Therapie (Statine bei Hypercholesterinämie, Antidiabetika bei bestehenden Diabetes Mellitus, Optimierung der antihypertensiven Medikation) noch spezifischer eingeleitet (Assmann et al. 1999) und zum anderen in interdisziplinärer Abstimmung fachübergreifende therapeutische oder diagnostische Maßnahmen wie Optimierung einer medikamentösen Herzinsuffizienztherapie oder Indikation zur Herzkatheter Untersuchung initiiert werden.

Des Weiteren zeigen die Ergebnisse, dass Patienten, bei denen eine eingeschränkte Pumpfunktion und Wandbewegungsstörungen durch die Echokardiographie diagnostiziert wurde, folgerichtig häufiger, im Vergleich zum Gesamtkollektiv, vor ihrem Schlaganfall Erkrankungen aus dem kardiovaskulären Formenkreis aufwiesen. Dazu zählen Pathologien wie die koronare 
Herzkrankheit, stattgefundene Myokardinfarkte, Herzinsuffizienz oder die periphere arterielle Verschlusskrankheit. In diesem Kollektiv waren zudem vornehmlich männliche $(74,1 \%)$ Patienten und sie wiesen im Vergleich einen schwereren Schlaganfall auf (NIHSS: 3 (2; 7)). Die Daten zeigen auch, dass Patienten aus dieser Gruppe ein erhöhtes Sterblichkeitsrisiko im Vergleich zum Gesamtkollektiv aufwiesen und daher unbedingt in den Fokus therapeutischer Bemühungen durch das zuständige ärztliche Personal genommen werden sollten.

Patienten, die ein persistierendes Foramen ovale aufwiesen, zeigten bis auf ein jüngeres Alter (70 $\pm 7,5$ ) keine anderen signifikanten Unterschiede. Der Trend zum jüngeren Alter deckt sich mit den Empfehlungen jüngster Leitlinien, die für Patienten mit einem persistierenden Foramen ovale und einem kryptogenen Schlaganfall einen interventionellen Verschluss empfehlen (Diener et al. 2018).

\subsection{Bewertung der diagnostischen und therapeutischen Wertigkeit}

Wie auch schon andere Studien (Harloff et al. 2006; Strandberg et al. 2002) zeigen die Ergebnisse

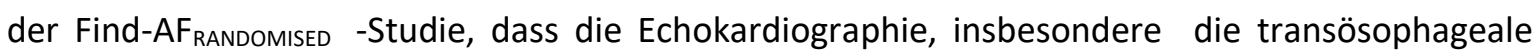
Variante, eine geeignete Methode darstellt, um mögliche kardioembolische Ursachen eines Schlaganfalls aufzudecken. So konnten überwiegend Pathologien diagnostiziert werden, die ein niedriges oder unklares Risiko zur Auslösung eines weiteren Schlaganfalls aufweisen (persistierendes Foramen ovale $n=33$, Vorhofseptumaneurysma $n=15$, Aortale Plaques $>4 \mathrm{~mm} n$ = 10). Befunde mit einem hohen Risiko für eine kardiogene Embolie fanden sich selten unter den Patienten dieser Studie, was möglicherweise auch im Zusammenhang mit dem Ausschluss der Patienten steht, die bereits ein Vorhofflimmern aufwiesen, da Patienten im Sinusrhythmus selten linksatriale Thromben aufweisen (Agmon et al. 2002).

Die transthorakale Echokardiographie ergab für die Patienten wenig neue Erkenntnisse über eine mögliche Ätiologie ihres Schlaganfalls (Mitralprolaps $n=4$, linksventrikuläres Aneurysma $n=1$, mindestens mittelgradige Aortenklappenstenose $\mathrm{n}=8$ ). Die Unterlegenheit zu der transösophagealen Variante diesbezüglich ist hinlänglich bewiesen (Sansoy et al. 1995; Doufekias et al. 2008). Hierbei muss jedoch berücksichtigt werden, dass die Überlegenheit der transöphagealen Echokardiographie in der Detektion möglicher Emboliequellen maßgeblich auf die große Anzahl von diagnostizierten persistierenden Foramen ovale zurückzuführen ist. Zu berücksichtigen ist, dass ein persistierendes Foramen ovale für die Allgemeinbevölkerung (Homma et al. 2016) keinen gesicherten Risikofaktor für zukünftige Schlaganfälle darstellt 
(Meissner et al. 2006) und kein etabliertes Therapiekonzept für Patienten, die ein Lebensalter über 60 Jahre aufweisen, bei der Diagnose besteht (Diener et al. 2018).

Eine breite Anwendung der transthorakalen Echokardiographie unter dem Gesichtspunkt der ätiologischen Einordnung des Schlaganfalls sollte daher in Frage gestellt werden. Vielmehr liefert die transthorakale Echokardiographie Erkenntnisse über den kardiologischen Status des Patienten und damit verbundene Pathologien (LVEF $<55 \% n=42$, Wandbewegungsstörungen/Akinesien $n=$ 44). Interessanterweise wurde in dieser Studie beobachtet, dass wenige dieser Befunde eine Erwähnung im Entlassungsbrief des behandelnden Neurologen finden. Den vielen Untersuchungen und dem damit verbundenen Aufwand und der Investition von Ressourcen steht somit wenig Ertrag für den Patienten gegenüber. Sehr selten konnte eine Empfehlung zum weiteren Prozedere wie z. B. weiterführende Untersuchungen im ambulanten Bereich durch die kardiologischen Kollegen während des stationären Aufenthalts beobachtet werden, wenn in der Echokardiographie ein relevanter Befund erhoben wurde. Da, wie in Kapitel 1.4 bereits dargestellt, die Erkenntnisse über diese Befunde jedoch die Prognose des Patienten beeinflussen, sollte hier mehr Aufmerksamkeit dem echokardiographischen Befund zuteilwerden. Mit dem bereits beschriebenen Hintergrund, dass ein Schlaganfall ein wichtiges Ereignis darstellt, um Patienten als kardiovaskulären Risikopatienten zu identifizieren, unterstreichen diese Erkenntnisse die Bedeutung einer Optimierung der Kooperation zwischen den Fachrichtungen Neurologie und Kardiologie.

Weiterhin zeigt sich als Auffälligkeit in der Studie, dass die teilnehmenden Studienzentren jeweils unterschiedliche therapeutische Ansätze bezüglich der Behandlung eines neu diagnostizierten persistierenden Foramen ovale anwendeten. Diese Behandlungen umfassten Strategien wie die medikamentöse Therapie mit ASS 300 mg, die Umstellung von ASS 100 mg auf Clopidogrel 75mg, die Einleitung einer oralen Antikoagulation bei wiederholenden Schlaganfällen. In einigen Fällen wurde eine orale Antikoagulation als empirische medikamentöse Therapie auf Grund eines individuellen Ermessens der behandelnden Ärzte eingeleitet, da mit dem Hintergrund der vorliegenden Befunde das persistierende Foramen ovale die wahrscheinlichste ätiologische Begründung für den Schlaganfall des Patienten darstellte. Der interventionelle Verschluss mittels

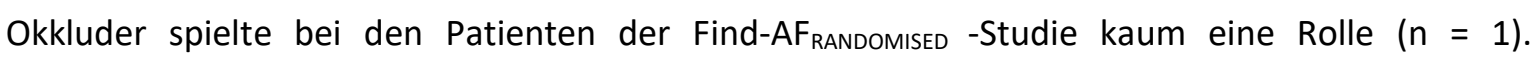
Interessanterweise wurde in einem Studienzentrum häufiger die Empfehlung zum interventionellen Verschluss eines persistierenden Foramen ovale durch den kardiologischen Untersucher gestellt, jedoch folgte daraus lediglich in einem Fall durch den behandelnden Neurologen die Empfehlung, diesen Eingriff im Anschluss an den Krankenhausaufenthalt durchzuführen. Diese Beobachtungen zeigen die Unsicherheit in der klinischen Praxis, welche zum 
Zeitpunkt der Durchführung der Find-AF $F_{\text {RANDOMISED }}$-Studie bezüglich der Behandlung eines persistierenden Foramen ovale bestand, was vermutlich auch durch die wechselnden Erkenntnisse der letzten Jahre bedingt ist. Die zunächst vorherrschende Meinung, dass der interventionelle Verschluss eines persistierenden Foramen ovale der medikamentösen Behandlung nicht überlegen zu sein scheint (Meier et al. 2013; Furlan et al. 2012; Carroll et al. 2013), wurde kurz darauf in Frage gestellt (Mas et al. 2017). Hier gibt es nun neue Erkenntnisse in Form einer neu veröffentlichten Leitlinie, welche einen interventionellen Verschluss für Patienten < 60 Jahre und einem kryptogenen Hirninfarkt empfiehlt (Diener et al. 2018), wodurch sicherlich ein einheitlicheres Vorgehen in Zukunft zu erwarten ist. Einen Graubereich stellt jedoch weiterhin, auch nach Veröffentlichung der Leitlinie, die Behandlung eines persistierenden Foramen ovale als mögliche Emboliequelle bei Patienten, die ein Lebensalter über 60 Jahre aufweisen dar, da hier entsprechende Daten für Empfehlungen seitens der Fachgesellschaften fehlen (Diener et al. 2018).

Mit diesem Hintergrund sind daher rückblickend einzelne Fälle dieser Studie, bei denen es zu der Einleitung einer oralen Antikoagulation aufgrund eines persistierendes Foramen ovale als kardiale Emboliequelle gekommen ist, kritisch zu hinterfragen. Bei einer klaren Anwendung der aktuellen Leitlinien wäre die Anzahl der Indikationsstellungen für die orale Antikoagulation (keine ausreichende Evidenz für einen Vorteil einer oralen Antikoagulation im Vergleich zur Thrombozytenaggregationshemmung (Diener et al. 2018)) geringer gewesen, womit letztendlich die therapeutische Wertigkeit der transösophagealen Echokardiographie, auch in dieser Arbeit, niedriger ausgefallen wäre. Dieses Problem beträfe auch die im Kapitel 4.1. vorgestellten Studien, welche die klinische Wertigkeit der transösophagealen Echokardiographie hauptsächlich an der Anzahl der Indikationen zur oralen Antikoagulation messen (Harloff et al. 2006; Warner 1996; Katsanos et al. 2016; Rauh et al. 1996; Strandberg et al. 2002). Schlussfolgernd ist daher davon auszugehen, dass die neu erschienene Leitlinie zur Behandlung eines persistierenden Foramen ovale die Wertigkeit der transösophagealen Echokardiographie für Patienten, die jünger als 60 Jahre sind und einen kryptogenen Schlaganfall erlitten haben, stärkt, da für diese Patienten ein interventioneller Verschluss nun evidenzbasiert empfohlen wird.

Auch andere, durch die transösophageale Echokardiographie in der Find-AF RANDOMISED $_{\text {-Studie }}$ diagnostizierte Pathologien ließen interessante Rückschlüsse zu. Der Zusammenhang aortaler Plaques, welche eine Größe von mehr als vier Millimeter aufweisen, und einem erhöhten Risiko einen Schlaganfall zu erleiden, ist hinlänglich bekannt (Amarenco et al. 1994). Der optimale therapeutische Ansatz zur Sekundärprophylaxe befindet sich jedoch noch im wissenschaftlichen Diskurs (Amarenco et al. 2014). Auch wenn die Studie von Amarenco et al. (2014) bezüglich dieser 
Thematik abgebrochen werden musste bevor die Zahlen statistische Wertigkeit erreicht haben, zeichnete sich ein Vorteil von einer Therapie mit plättchenhemmenden Wirkstoffen (Clopidogrel und Aspirin) im Gegensatz zu Marcumar ab. Die Diagnostik von aortalen Plaques ist demzufolge in der Routine nicht empfohlen, da es zum jetzigen Zeitpunkt keine Auswirkung auf die Therapie des Patienten hat (Yang et al. 2016). Dies unterstreicht die momentan untergeordnete Rolle aortaler Plaques als potentielle Emboliequelle eines Hirninfarkts. Zukünftig könnte diese Pathologie in der Diagnostik des ischämischen Schlaganfalls noch weiter in den Hintergrund geraten, da gezeigt wurde, dass durch den Einsatz einer hochdosierten Therapie mit Statinen bei Patienten, die ein hohes Risiko für kardiovaskuläre Ereignisse aufweisen, atherosklerotische Komplikationen deutlich reduziert werden konnten (Amarenco et al. 2006; Sever et al. 2003). Patienten der Find$\mathrm{AF}_{\text {RANDOMISED }}$-Studie mit nachgewiesenen aortalen Plaques größer vier Millimeter erhielten keine Änderung in der Therapie.

Ähnliches ließ sich auch bei anderen Pathologien wie dem spontanen Echo-Kontrast, Mitralklappenprolaps oder der Aortenstenose beobachten. Auch wenn für diese Diagnosen noch kein eindeutiger kausaler, ätiologischer Zusammenhang zum Schlaganfall bewiesen ist (Yang et al. 2016) und es daher zum jetzigen Zeitpunkt ungewiss ist, ob eine Änderung der bestehenden Sekundärprophylaxe einen Vorteil für den Patienten ergibt (Yang et al. 2016), sind einige dieser Pathologien mit einem niedrigen jährlichen Risiko von unter $2 \%$ einen Schlaganfall zu erleiden assoziiert (Ay et al. 2005). Auch diese Diagnosen führten, wenn auch im Befund durch den Kardiologen erwähnt, zu keiner therapeutischen Konsequenz bei den Find-AFRANDOMISED -Patienten.

Somit unterstützen die Ergebnisse dieser Arbeit auch den aktuellen Wissensstand. Denn entscheidend für den Einsatz einer diagnostischen Maßnahme ist es, ob die neuen Erkenntnisse auch therapeutische Konsequenzen für den Patienten ermöglichen (Morris et al. 2009). Hier zeigt sich, dass viele Befunde in der Echokardiographie, insbesondere der transösophagealen Echokardiographie (Yang et al. 2016), nicht zu einer Änderung in der Therapie führen (Morris et al. 2009). Dieses gilt vor allem aus den bereits beleuchteten Gründen für Patienten, die ein Lebensalter über 60 aufweisen. Diagnosen sind durch die Vorbefunde bereits bekannt, durch andere Maßnahmen im Vorfeld identifiziert worden (z. B. Vorhofflimmern in der Telemetrie (Morris et al. 2009) und haben so bereits zur Indikationsstellung einer oralen Antikoagulation geführt. Ist der Patient bereits antikoaguliert, ergibt sich aus derzeitiger Sicht (mit Ausnahme eines konkreten klinischen Verdachts auf eine Endokarditis) keine Notwendigkeit für weitere ätiologische Diagnostik, da so bereits alle in Frage kommenden Pathologien therapeutisch abgedeckt sind (Warner 1996). Da für einige Pathologien (z. B. aortale Plaques) bisher noch keine Wirksamkeit einer spezifischen therapeutischen Strategie im Vergleich zur standardisierten, 
leitliniengerechten Therapie nach stattgefundenem Hirninfarkt (DGN 2015) nachgewiesen werden konnte, haben nur wenige Befunde der Echokardiographie eine Konsequenz für den Patienten. Eine Ausnahme stellt die Empfehlung zum interventionellen Verschluss eines persistierenden Foramen ovale bei Patienten < 60 Jahre (Diener et al. 2018) dar.

Auch der in dieser Arbeit angefertigte Vergleich der Echokardiographie mit einer Langzeit-EKGUntersuchung anhand der Anzahl therapeutischer Konsequenzen für den Patienten bietet eine Möglichkeit die Wertigkeit der Echokardiographie zu beurteilen. Zunächst ist zu erwähnen, dass dieser Vergleich sich nur auf die Anzahl therapeutischer Änderungen beschränkt und keinen grundlegenden Vergleich dieser beiden diagnostischen Methoden darstellen soll. Die LangzeitEKG-Untersuchung mittels Holter Monitor über sieben Tage bewies sich als kosteneffektiv (Mayer et al. 2013) und, wenn über zehn Tage angewendet, sehr effektiv in der Diagnostik eines möglichen paroxsymalen Vorhofflimmerns als ätiologische Ursache eines Schlaganfalls (Wachter et al. 2017). Der im Kapitel 3.5.1 aufgeführte Vergleich zeigt, dass die zehn Tage Langzeit-EKGUntersuchung nicht nur eine gute diagnostische Methode zur ätiologischen Abklärung darstellt, mehr noch führt sie häufiger in Form einer oralen Antikoagulation zu einer therapeutischen Konsequenz (Rate an Intervention Echokardiographie 4,74\%; Rate an Interventionen Langzeit EKG: 13,5 \%). Im Gegensatz zu einer Mehrzahl der therapeutischen Konsequenzen aus den Befunden der Echokardiographie, entspricht diese Therapie zudem den Empfehlungen aktueller Leitlinien (Kirchhof et al. 2016; January et al. 2019). Hierbei sei jedoch zu erwähnen, dass der Nutzen einer oralen Antikoagulation zur Primärprophylaxe eines Hirninfarkts bei Patienten mit einem Vorhofflimmern unabhängig von seiner Präsentation (permanent oder paroxysmal, diagnostiziert mit einem 12-Kanal EKG) nachgewiesen wurde (Hohnloser et al. 2007; Friberg et al. 2010), die gleichwertige Wirksamkeit zur Sekundärprophylaxe für kurze Episoden eines paroxysmalen Vorhofflimmerns, welches im Langzeit-Holter EKG nachgewiesen wurde, formal aussteht (Wachter et al. 2013).

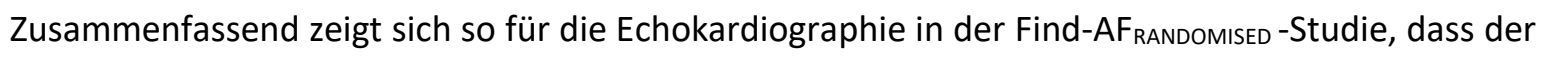
Anteil von therapeutischen Konsequenzen im Verhältnis zu den gestellten Diagnosen sehr gering ist. Eine empfohlene Quote, 15\% aller Stroke Unit Patienten einer transösophagealen Echokardiographie zu unterziehen, wie es in den aktuellen Zertifizierungskriterien der Deutschen Schlaganfallgesellschaft für Stroke Units empfohlen wird (Nabavi et al. 2015), ist somit zumindest kritisch zu hinterfragen. Mit dem Hintergrund des Risikos einer invasiven Methode und einer nicht gesicherten Kosten-Effektivität (Meenan et al. 2007) ist vor allem bei der transösophagealen Untersuchung eine strengere Indikationsstellung sinnvoll, zumal bei einer nicht unerheblichen Anzahl der Patienten, bei denen eine ätiologische Einordung des Hirninfarkts zunächst nicht 
gelungen ist, ein paroxysmales Vorhofflimmern besteht, das durch die Standard Rhythmus Kontrolle nicht diagnostiziert werden konnte (Wachter et al. 2017). Die Durchführung einer transösophagealen Echokardiographie sollte sich daher strenger an klaren klinischen Fragestellungen, wie beispielsweise ein klinischer Verdacht auf Endokarditis, rezidivierende Schlaganfälle unbekannter Ursache, embolisches Infarktmuster in der zerebralen Bildgebung, juvenile Hirninfarkte oder Klappenvitien, die nicht ausreichend in einer thransthorakalen Echokardiographie zu beurteilen sind, orientieren. Zudem nimmt die Bedeutung einer transösophagealen Echokardiographie für Patienten, die ein Lebensalter über 60 Jahre aufweisen, nach der Empfehlung zum internventionellen Verschluss eines persistierenden Foramen ovale für diese Patientengruppe deutlich zu (Diener et al. 2018). Die Indikationsstellung zu einer thransthorakalen Echokardiographie kann hingegen großzügig erfolgen. Nicht, wie bereits beschrieben, zwecks ätiologischer Einordnung des Schlaganfalls, sondern vielmehr, um ein möglichst breites kardiovaskuläres Risikoprofil des Patienten zu erstellen.

\subsection{Methodische Einordnung und Limitationen der Studie}

Da es sich bei der Find-AF RANDOMISED -Studie um eine kontrollierte, randomisierte und multizentrische Studie handelt, weisen die Daten eine hohe Güte auf. Die Daten zur Echokardiographie wurden standardisiert anhand eines CRF-Bogens (siehe Anhang) in jedem Studienzentrum prospektiv aufgenommen, wodurch eine einheitliche Datengewinnung garantiert werden konnte und dadurch ein Vorteil gegenüber retrospektiven Metaanalysen (Katsanos et al. 2016) besteht.

Kritisch zu erwähnen ist, dass die Beurteilung echokardiographischer Konsequenzen keine prädefinierte Fragestellung der Find- $\mathrm{AF}_{\mathrm{RANDOMISED}}$-Studie darstellte, sondern vielmehr zum Ziel hatte, die Rhythmus-Diagnostik von Schlaganfall-Patienten mittels einer prolongierten LangzeitEKG-Untersuchung zu untersuchen. Die Echokardiographie stand somit nicht im Fokus der Studie. Dieser Umstand resultierte in einer geringen Anzahl an transösophagealen Echokardiographien , gemessen an der Patientenzahl, mit vergleichbaren Studien (Harloff et al. 2006; Strandberg et al. 2002). Als häufigster Grund für eine nicht durchgeführte transösophageale Echokardiographie wurde die nicht vorhandene Kapazität angeführt ( $n=146)$, was nochmals den hohen Ressourcenaufwand dieser Untersuchung in der klinischen Routine verdeutlicht. Zudem erfolgte die Echokardiographie in den Studienzentren im Rahmen der klinischen Routine und nicht durch speziell zertifizierte Untersucher. Hierdurch wird nicht nur die Kontrolle der Daten erschwert, auch die Qualität der Untersuchungen wird durch die Vielzahl an Untersuchern kompromittiert. 
Diese Heterogenität hat jedoch auch den Vorteil, dass die Ergebnisse die klinische Realität im Krankenhaus Alltag optimal abbilden. Ein Studiendesign, welches auf die Echokardiographie ausgelegt gewesen wäre, hätte womöglich hier zu einer höheren Anzahl an Untersuchungen und damit zu einer höheren Aussagekraft geführt.

Ein weiterer Nachteil dieser Arbeit ergab sich durch die Einschlusskriterien, deren Festlegung sich verständlicherweise an den Fragestellungen zum paroxsymalen Vorhofflimmern und des 10 Tage Langzeit-EKG Holter Monitoring orientierte. Zwar erfolgte die Untersuchung der klinischen Wertigkeit nicht an Subgruppen wie „embolic stroke of undetermined source“ (ESUS) (Hart et al. 2014) wie bei Katsanos et al (Katsanos et al. 2016) oder der „stroke of undetermined etiology“ (Harloff et al. 2006), dennoch wurde durch die Einschlusskriterien ein spezielles Patientenkollektiv geschaffen.

Weiterhin wäre es interessant gewesen, die klinische Wertigkeit der Echokardiographie, insbesondere der transösophagealen Variante, bei jüngeren Patienten zu untersuchen. Möglicherweise profitieren gerade jüngere Patienten durch eine transösophageale Echokardiographie (Rus Mansilla et al. 2008). Das gewählte Alter von mehr als 60 Jahren ermöglicht jedoch eine bessere Übertragung der Ergebnisse auf Patienten der Gesamtbevölkerung, die an kardiovaskulären Erkrankungen leiden, da diese in der Regel ein höheres Lebensalter aufweisen (Assmann et al. 1999).

\subsection{Klinische Relevanz und Ausblick}

Auch die Ergebnisse dieser Studie haben gezeigt, dass die Echokardiographie ein wichtiges diagnostisches Werkzeug darstellt, um neue Erkenntnisse über den Patienten zu gewinnen.

Viel wichtiger sind jedoch die Konsequenzen, die sich daraus ergeben. In den Zeiten von steigender Rationalisierung und der damit verbundenen Optimierung der Ressourcenverteilung könnte mit Hilfe eines geeigneten Modells eine Vorhersage getroffen werden, welche Patientengruppen vermehrt von dem Einsatz der Echokardiographie profitieren könnten. Eine solche Selektion könnte möglichweise Ressourcen einsparen, die in anderen Bereichen, wie beispielsweise der Detektion von paroxysmalem Vorhofflimmern mittels Langzeit-EKG, dringend benötigt werden. Eine Folge wäre, dass Patienten, die eine geringe Wahrscheinlichkeit für Befunde in der Echokardiographie aufweisen, diese Untersuchung im ambulanten Bereich durchführen lassen können, um den kostenintensiven Krankenhausaufenthalt zu verkürzen (Menon et al. 2014). 
Ein weiteres Modell zur Selektion besteht darin, die transthorakale Echokardiographie als Screening Methode zur Auswahl der Patienten, die eine transösophageale Echokardiographie bekommen sollen, einzusetzen. Patienten mit einer befundlosen transthorakalen Echokardiographie weisen weniger potentielle kardiale Emboliequellen, wie etwa spontanen Echo Kontrast, aortale Plaques oder Thromben in der nachfolgenden transösophagealen Echokardiographie auf (Leung et al. 1995). Zudem besteht die Möglichkeit, die transthorakale Echokardiographie zur Screening Methode für größere intra-atriale Shunts infolge eines persistierenden Foramen ovale zu etablieren. Daniëls et al. (2004) zeigten in dieser Hinsicht eine vergleichbare Sensitivität zur Detektion eines persistierenden Foramen ovale im Vergleich zur Untersuchung mittels transösophagealer Echokardiographie, welche den aktuellen etablierten Gold-Standard darstellt. Hierdurch könnte eine weitere Reduktion der Indikationsstellung zur transöphagealen Echokardiographie erreicht werden.

Die Tatsache, dass die Veröffentlichung dieser Studien schon Jahre zurückliegt, unterstreicht jedoch, dass bis heute verlässliche Daten zur optimalen Behandlung einiger kardioembolischer Quellen fehlen. Diese Daten stellen jedoch die Grundlage zur Entwicklung eines verlässlichen Modells zur Selektion der Patienten, die eine Echokardiographie erhalten sollten, dar. Dies wird auch durch die Veröffentlichung der Leitlinie zur Behandlung eines persistierenden Foramen ovale als mögliche Emboliequelle deutlich. Diese gibt nur Empfehlungen für Patienten, die ein Lebensalter unter 60 Jahren aufweisen. Die Behandlung von Patienten außerhalb dieser Altersgrenze bleibt, auf Grund mangelnder Datenlage, ein Graubereich (Diener et al. 2018). Zudem werden in den kommenden Jahren die Ergebnisse großer Studien zum Management von ESUS-Patienten (Geisler et al. 2017; Kamel et al. 2019), die eine immer zentralere Rolle entwickeln werden, wichtige Erkenntnisse erbringen.

Eine weitere wichtige Erkenntnis für die klinische Arbeit betrifft die interdisziplinäre Zusammenarbeit zwischen den neurologischen und den kardiologischen Abteilungen. Die Bedeutung einer effektiven Zusammenarbeit dieser beiden Fachabteilungen wird durch die hohe Anzahl kardialer Komplikationen wie z. B. einem plötzlichen Herztod (Sörös und Hachinski 2012) nach einem Schlaganfall untermauert (Chen et al. 2017). Auch wenn die genauen pathophysiologischen Zusammenhänge und die prognostische Relevanz der Interaktion zwischen den beiden Organsystemen nicht abschließend geklärt ist, wird vermutet, dass akute Schlaganfälle durch eine autonome Dysregulation die Herzfunktion entscheidend beeinflussen können (Sörös und Hachinski 2012).

Es zeigte sich, dass in vielen Fällen erhobene Befunde durch den Kardiologen, die auf den ersten Blick keinen ätiologischen Zusammenhang mit dem Schlaganfall aufwiesen, nicht durch den 
behandelnden Neurologen berücksichtigt wurden. Darunter fielen Befunde wie Klappenvitien oder Wandbewegungsstörungen. Die aus diesen Befunden resultierenden Konsequenzen wie z. B. eine Koronarangiographie, eine Optimierung der antihypertensiven Medikation oder eine Überweisung zu einem ambulanten Kardiologen blieben so häufig aus. Dabei zeigt sich mit dem Hintergrund, dass die Ätiologie eines Schlaganfalls sich bei einem weiteren Ereignis in 45,5\% der Fälle ändert (Hillen et al. 2003), dass der erste Schlaganfall als dringende Aufforderung an den behandelnden Arzt gesehen werden sollte, eine möglichst breite kardiovaskuläre Diagnostik einzuleiten, da diese auch die Prognose des Patienten deutlich mitbestimmt. Im klinischen Alltag hingegen zielt die eingeleitete Diagnostik vor allem darauf ab, eine Ursache des Hirninfarkts zu identifizieren. Zeigt sich beispielsweise mittels Duplex-Sonographie eine schwere Atheromatose der hirnversorgenden Arterien als arteriell-embolische (makroangiopathisch nach TOAST) Ursache eines Hirninfarkts, werden weitere diagnostische Bemühungen eingestellt. Wie auch die Ergebnisse dieser Arbeit andeuten (z. B. erhöhte Anzahl von Wandbewegungsstörungen bei Patienten, die bereits im Vorfeld kardiovaskulär erkrankt sind), profitieren womöglich diese Patienten von weiterer Diagnostik wie einer transthorakalen Echokardiographie oder eines verlängerten EKG-Monitorings, um das Risiko kardiovaskulärer Ereignisse anderer Identität, wie einem akuten Koronarsyndrom, oder einem Schlaganfall-Rezidiv infolge eines bisher unbekannten paroxysmalen Vorhofflimmerns, zu verhindern.

Konkret sollte der Einsatz der Echokardiographie in der Diagnostik des ischämischen Schlaganfalls daher nicht zur reinen ätiologischen Einteilung verstanden werden, sondern ebenfalls Hinweise auf den kardiologischen Status des Patienten liefern, denen konsequent nachgegangen werden sollte, da Krankheiten wie die periphere arterielle Verschlusskrankheit oder die koronare Herzerkrankung gleiche Risikofaktoren wie die eines Hirninfarkts aufweisen (Bhatt et al. 2006). Bekräftigt wird diese Argumentation dadurch, dass die Mortalität von Patienten, die einen Hirninfarkt erlitten haben, entscheidend durch das Vorhandensein einer koronaren Herzkrankheit beeinflusst wird (Brønnum-Hansen et al. 2001).

Abbildung 6 zeigt einen Vorschlag zur Durchführung einer Echokardiographie nach ischämischen Hirninfarkt: 
Abbildung 6: Echokardiographische Diagnostik nach ischämischem Schlaganfall

Ischämischer Schlaganfall oder TIA

Initiale Akutdiagnostik

- Vorerkrankungen, Kontraindikationen gegen orale Antikoagulation

- Klinisch-neurologische Untersuchung

- Blutentnahme und laborchemische Untersuchung, 12 Kanal EKG

- Zerebrale Bildgebung (CT/MRT)

- Gefäßdartellung: Extra und transkranielle Duplex-Sonographie, ggf. CTA/MRA

- Überwachung der Vitalparameter, Telemetrie 24-72 h

Ätiologischer Nachweis gelungen*

- Kardiovaskuläres Risikoprofil

- Atherosklerose in der Duplex-Sonographie

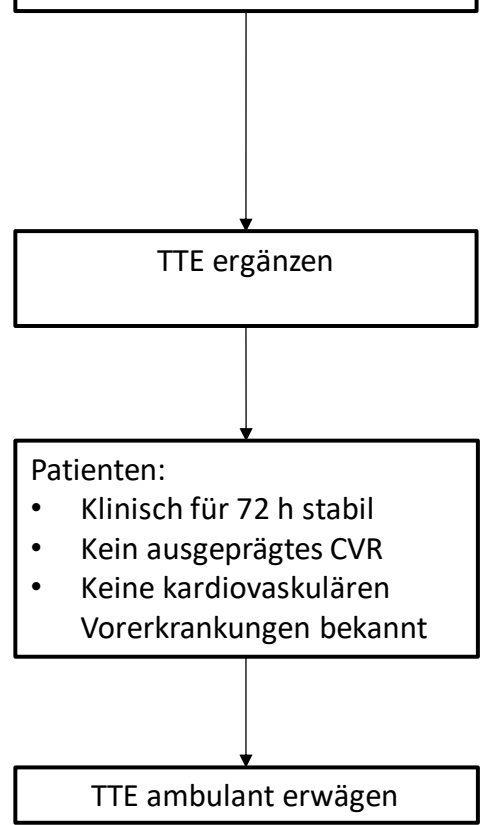

Kein ätiologischer Nachweis gelungen
- Juveniler Hirninfarkt

- Patienten < 60 Jahre

- Wiederholte ESUS oder Hirninfarkte unbekannter Ursache

- Verdacht auf Endokarditis

- Embolisches Verteilungsmuster in der zerebralen Bildgebung

TTE gefolgt von TEE

*: Ätiologischer Nachweis gelungen: Nachweis von VHF, Nachweis arteriell-embolischer Emboliequelle, signifikante Gefäßstenose, Bildgebend lakunärer Hirninfarkt, Nachweis spezieller Ursachen (z. B. Dissektion, Vaskulitis). 


\section{Zusammenfassung}

In der Behandlung von Patienten, die einen akuten Schlaganfall erlitten haben, nimmt die Diagnostik zur ätiologischen Einordung dieses Krankheitsbildes eine wichtige Rolle ein. Die Echokardiographie ist hierbei neben dem Langzeit-EKG Mittel der Wahl in der Detektion möglicher kardioembolischer Ursachen und ist im klinischen Alltag als quantitativ häufig eingesetzte Methode präsent. Ziel dieser Arbeit ist es, mit Hilfe von Daten aus der multizentrischen, randomisierten und kontrollierten Find-AFRANDOMISED -Studie die klinische Wertigkeit der thransthorakalen und transösophagealen Echokardiographie bei Schlaganfall-Patienten zu untersuchen.

Es zeigte sich, dass neben der Bestimmung mutmaßlicher Ursachen für den stattgehabten Hirninfarkt, die Wertigkeit dieser Methoden vielmehr durch die Erfassung potentiell Prognose relevanter kardiovaskulärer Begleiterkrankungen bestimmt wird. Dies gilt insbesondere dann, wenn die erfassten Befunde eine unmittelbare Konsequenz für die weitere Behandlung der Patienten nach sich zogen.

Letztendlich erbrachte diese Arbeit, dass die Echokardiographie zwar viele kardiovaskuläre Befunde aufdeckt, diese jedoch selten zu therapeutischen Konsequenzen bei den Patienten führen. Diese niedrige Anzahl ist vor allem Folge fehlender Evidenz zur Behandlung der diagnostizierten Pathologien, sowie ausbleibender interdisziplinärer Zusammenarbeit. Um einen höheren Nutzen für den Patienten zu erreichen, ist eine Verbesserung der interdisziplinären Arbeit zwischen Neurologen und Kardiologen notwendig, um fachübergreifende Therapien und Diagnostik einzuleiten. Ein Schlaganfall sollte daher als ein Indexereignis für den Patienten verstanden werden, um möglichst breite, multidisziplinäre, kardiovaskuläre Diagnostik einzuleiten, um weitere Erkrankungen aus diesem Formenkreis zu identifizieren und folgende Ereignisse gezielt zu verhindern, da bestehende Risikofaktoren sich hier häufig überschneiden.

Einen hohen Stellenwert dieser kardiovaskulären Diagnostik bei Schlaganfall-Patienten stellt die transthorakale Echokardiographie dar. Die Indikation hierzu sollte großzügig erfolgen. Der Einsatz der transösophagealen Echokardiographie hingegen sollte sich gezielt an definierte Kriterien wie dem Alter oder konkretem klinischen Verdacht auf zugrundeliegende Pathologien richten. Die so rekrutierten Ressourcen können dadurch in andere diagnostische Methoden, wie beispielsweise einer Langzeit-EKG-Untersuchung investiert werden, welche im gezeigten Vergleich mit der Echokardiographie eine hohe therapeutische Wertigkeit im Bezug zur Indikationsstellung therapeutischer Konsequenzen besitzt. 


\section{Anlagen}

Abbildung 7: CRF TTE Untersuchung der Find-AF RANDOMISED $_{\text {-Studie }}$

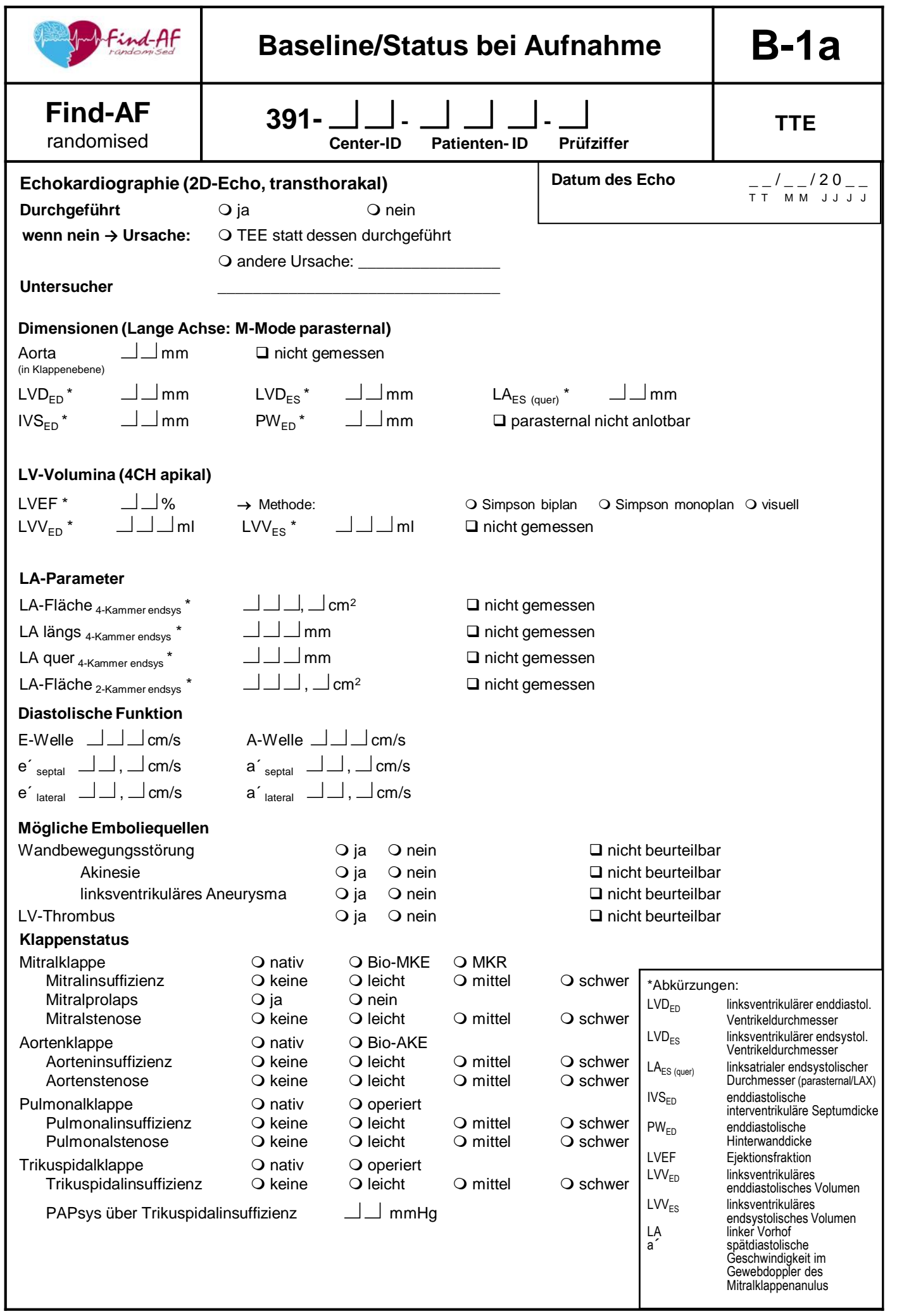




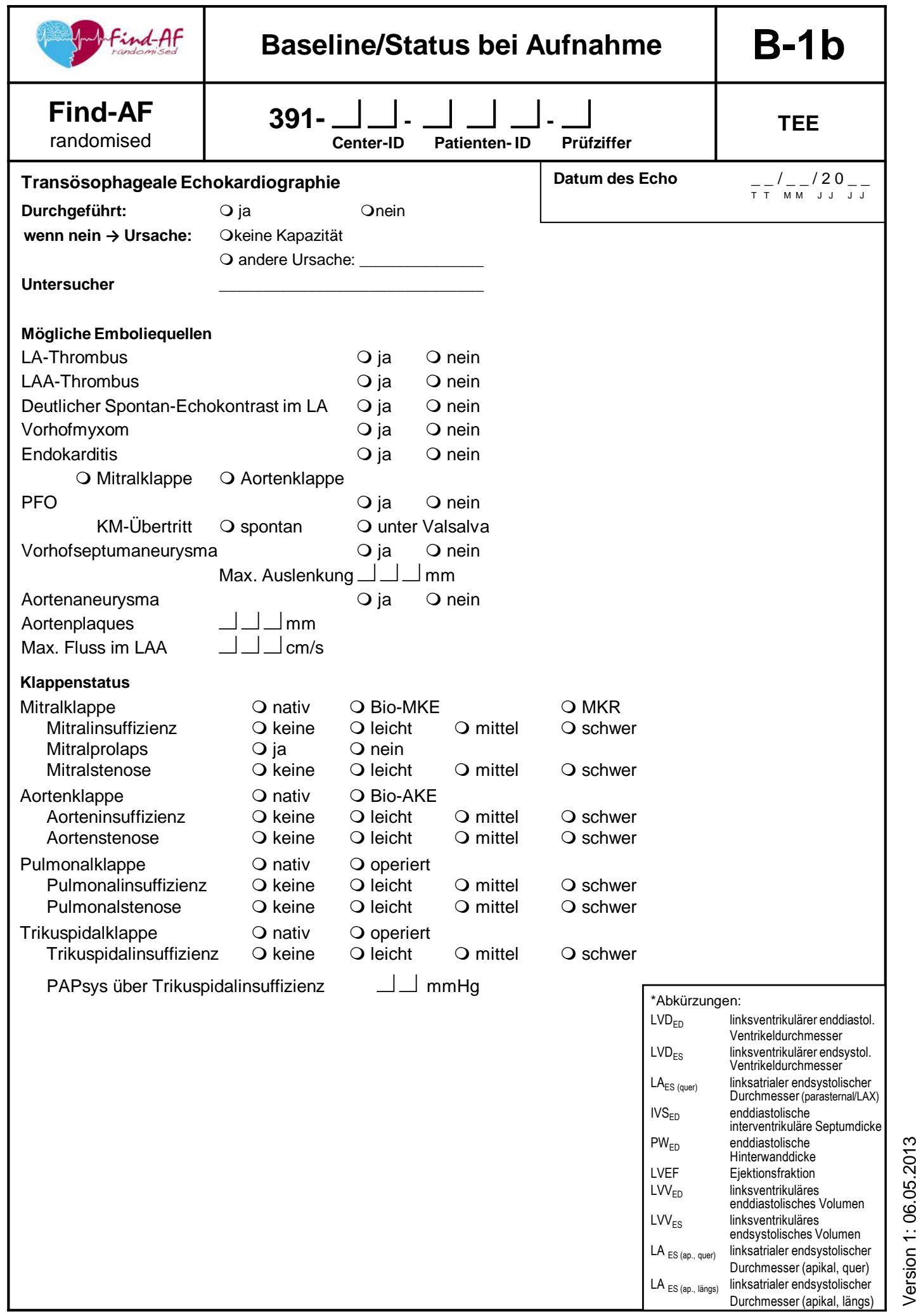




\section{Literaturverzeichnis}

\section{Literaturverzeichnis}

Adams HP, Bendixen BH, Kappelle L, Biller J, Love BB, Gordon DL, Marsh EE (1993): Classification of subtype of acute ischemic stroke. Definitions for use in a multicenter clinical trial. TOAST. Trial of Org 10172 in Acute Stroke Treatment. Stroke 24, 35-41

Agmon Y, Khandheria BK, Gentile F, Seward JB (2002): Clinical and echocardiographic characteristics of patients with left atrial thrombus and sinus rhythm: experience in 20643 consecutive transesophageal echocardiographic examinations. Circulation 105, 27-31

Albers GW, Caplan LR, Easton JD, Fayad PB, Mohr JP, Saver JL, Sherman DG (2002): Transient ischemic attack-proposal for a new definition. N Engl J Med 347, 1713-1716

Amarenco P, Bogousslavsky J, Callahan A, Goldstein LB, Hennerici M, Rudolph AE, Sillesen H, Simunovic L, Szarek M, Welch KMA et al. (2006): High-dose atorvastatin after stroke or transient ischemic attack. N Engl J Med 355, 549-559

Amarenco P, Cohen A, Tzourio C, Bertrand B, Hommel M, Besson G, Chauvel C, Touboul PJ, Bousser MG (1994): Atherosclerotic disease of the aortic arch and the risk of ischemic stroke. N Engl J Med 331, 1474-1479

Amarenco P, Davis S, Jones EF, Cohen AA, Heiss WD, Kaste M, Laouénan C, Young D, Macleod M, Donnan GA (2014): Clopidogrel plus aspirin versus warfarin in patients with stroke and aortic arch plaques. Stroke $\underline{45}, 1248-1257$

Arboix A, Alió J (2010): Cardioembolic stroke: clinical features, specific cardiac disorders and prognosis. Curr Cardiol Rev $\underline{6}, 150-161$

Arboix A, Martí-Vilalta JL (2009): Lacunar stroke. Expert Rev Neurother $\underline{9}$, 179-196

Assmann G, Cullen P, Jossa F, Lewis B, Mancini M (1999): Coronary heart disease: reducing the risk: the scientific background to primary and secondary prevention of coronary heart disease. $\mathrm{A}$ worldwide view. International task force for the prevention of coronary heart disease.

Arterioscler. Thromb. Vasc. Biol. 19, 1819-1824

Astrup J, Siesjö BK, Symon L (1981): Thresholds in cerebral ischemia - the ischemic penumbra. Stroke $12,723-725$

Ay H, Furie KL, Singhal A, Smith WS, Sorensen AG, Koroshetz WJ (2005): An evidence-based causative classification system for acute ischemic stroke. Ann. Neurol. $\underline{58}, 688-697$

Bähr M, Frotscher M: Neurologisch-topische Diagnostik: Anatomie ; Funktion ; Klinik, 10. Auflage; Georg Thieme Verlag, Stuttgart 2014

Bhatt DL, Steg PG, Ohman EM, Hirsch AT, Ikeda Y, Mas JL, Goto S, Liau CS, Richard AJ, Rother J et al. (2006): International prevalence, recognition, and treatment of cardiovascular risk factors in outpatients with atherothrombosis. JAMA $\underline{295}, 180-189$

Brønnum-Hansen H, Davidsen M, Thorvaldsen P (2001): Long-term survival and causes of death after stroke. Stroke 32, 2131-2136 
Busse O, Darius H (2002): Indikationen zur Echokardiographie beim ischämischen zerebralen Insult. Nervenarzt $\underline{73}, 792-795$

Camm AJ, Kirchhof P, Lip GYH, Schotten U, Savelieva I, Ernst S, van Gelder IC, Al-Attar N, Hindricks $G$, Prendergast B et al. (2010): Guidelines for the management of atrial fibrillation: the task force for the management of atrial fibrillation of the European Society of Cardiology (ESC). Eur Heart J 31, 2369-2429

Carroll JD, Saver JL, Thaler DE, Smalling RW, Berry S, MacDonald LA, Marks DS, Tirschwell DL (2013): Closure of patent foramen ovale versus medical therapy after cryptogenic stroke. N Engl J Med 368, 1092-1100

Chen Z, Venkat P, Seyfried D, Chopp M, Yan T, Chen J (2017): Brain-heart interaction: cardiac complications after stroke. Circ Res $\underline{121}$, 451-468

Conway DSG, Pearce LA, Chin BSP, Hart RG, Lip GYH (2003): Prognostic value of plasma von Willebrand factor and soluble P-selectin as indices of endothelial damage and platelet activation in 994 patients with nonvalvular atrial fibrillation. Circulation 107, 3141-3145

Cotter PE, Martin PJ, Ring L, Warburton EA, Belham M, Pugh PJ (2013): Incidence of atrial fibrillation detected by implantable loop recorders in unexplained stroke. Neurology $80,1546-$ 1550

Coutts SB, Hill MD, Simon JE, Sohn CH, Scott JN, Demchuk AM (2005): Silent ischemia in minor stroke and TIA patients identified on MR imaging. Neurology $\underline{65}, 513-517$

Daniel WG, Erbel R, Kasper W, Visser CA, Engberding R, Sutherland GR, Grube E, Hanrath P, Maisch B, Dennig K (1991): Safety of transesophageal echocardiography. A multicenter survey of 10419 examinations. Circulation $\underline{83}, 817-821$

Daniëls C, Weytjens C, Cosyns B, Schoors D, J de Sutter, Paelinck B, Muyldermans L, van Camp G (2004): Second harmonic transthoracic echocardiography: the new reference screening method for the detection of patent foramen ovale. Eur J Echocardiogr $\underline{5}, 449-452$

Desai AJ, Fuller CJ, Jesurum JT, Reisman M (2006): Patent foramen ovale and cerebrovascular diseases. Nat Clin Pract Cardiovasc Med $\underline{3}$, 446-455

DGN (2015): S3 Leitlinie zur Sekundärprophylaxe ischämischer Schlaganfall und transitorische ischämische Attacke. http://www.dgn.org/images/red_leitlinien/LL_2014/PDFs_Download/030133_lang_S3_Sekund\%C3\%A4rprophylaxe_isch\%C3\%A4mischer_Schlaganfall_2015-02.pdf, abgerufen am: 06.11.2019

DGN (2017): S1 Leitlinie der deutschen Gesellschaft für Neurologie (DGN) zur Diagnostik akuter zerebrovaskulärer Erkrankungen.

https://www.dgn.org/images/red_leitlinien/LL_2017/PDFs_Download/030117_LL_Zerebrovaskul aere_Erkrankungen_2017.pdf, abgerufen am: 17.05.2019

Di Minno MND, Ambrosino P, Dello Russo A, Casella M, Tremoli E, Tondo C (2016): Prevalence of left atrial thrombus in patients with non-valvular atrial fibrillation. A systematic review and metaanalysis of the literature. Thromb. Haemost. 115, 663-677

Diener HC, Grau AJ, Baldus S, Ghanem A, Gröschel K, Liebetrau C, Massberg S, Möllmann H, Nef H, Sander D et al. (2018): Kryptogener Schlaganfall und offenes Foramen ovale S2e-Leitlinie.

Nervenarzt $\underline{89}, 1143-1153$ 
Doufekias E, Segal AZ, Kizer JR (2008): Cardiogenic and aortogenic brain embolism. J Am Coll Cardiol $\underline{51}, 1049-1059$

Easton JD, Saver JL, Albers GW, Alberts MJ, Chaturvedi S, Feldmann E, Hatsukami TS, Higashida RT, Johnston SC, Kidwell CS et al. (2009): Definition and evaluation of transient ischemic attack: a scientific statement for healthcare professionals from the American Heart Association/American Stroke Association Stroke Council; Council on Cardiovascular Surgery and Anesthesia; Council on Cardiovascular Radiology and Intervention; Council on Cardiovascular Nursing; and the Interdisciplinary Council on Peripheral Vascular Disease. The American Academy of Neurology affirms the value of this statement as an educational tool for neurologists. Stroke $\underline{40}, 2276-2293$

Eschenfelder CC, Zeller JA, Stingele R (2006): Schlaganfall: Ursachen und Klassifikation. Hamostaseologie 26, 298-308

Fisher M (1997): Characterizing the Target of Acute Stroke Therapy. Stroke 28, 866-872

Friberg L, Hammar N, Rosenqvist M (2010): Stroke in paroxysmal atrial fibrillation: report from the Stockholm Cohort of Atrial Fibrillation. Eur Heart J 31, 967-975

Furlan AJ, Reisman M, Massaro J, Mauri L, Adams H, Albers GW, Felberg R, Herrmann H, Kar S, Landzberg $\mathrm{M}$ et al. (2012): Closure or medical therapy for cryptogenic stroke with patent foramen ovale. N Engl J Med 366, 991-999

Geisler T, Poli S, Meisner C, Schreieck J, Zuern CS, Nägele T, Brachmann J, Jung W, Gahn G, Schmid E et al. (2017): Apixaban for treatment of embolic stroke of undetermined source (ATTICUS randomized trial): Rationale and study design. Int J Stroke 12, 985-990

Gladstone DJ, Spring M, Dorian P, Panzov V, Thorpe KE, Hall J, Vaid H, O'Donnell M, Laupacis A, Côté $R$ et al. (2014): Atrial fibrillation in patients with cryptogenic stroke. N Engl J Med $\underline{370}$, 24672477

Grau AJ, Weimar C, Buggle F, Heinrich A, Goertler M, Neumaier S, Glahn J, Brandt T, Hacke W, Diener HC (2001): Risk factors, outcome, and treatment in subtypes of ischemic stroke: the German stroke data bank: The German Stroke Data Bank. Stroke 32, 2559-2566

Haas, S. (Hrsg.) (2005): Prävention von Thrombosen und Embolien in der Inneren Medizin, 1. Auflage; Springer-Verlag, Berlin/Heidelberg 2005

Harloff A, Handke M, Reinhard M, Geibel A, Hetzel A (2006): Therapeutic strategies after examination by transesophageal echocardiography in 503 patients with ischemic stroke. Stroke 37, 859-864

Hart RG, Diener HC, Coutts SB, Easton JD, Granger CB, O'Donnell MJ, Sacco RL, Connolly SJ (2014): Embolic strokes of undetermined source: the case for a new clinical construct: The case for a new clinical construct. The Lancet Neurology $\underline{13}, 429-438$

Hart RG, Halperin JL, Pearce LA, Anderson DC, Kronmal RA, McBride R, Nasco E, Sherman DG, Talbert RL, Marler JR (2003): Lessons from the stroke prevention in atrial fibrillation trials. Ann Intern Med 138, 831-838

Heuschmann P, Busse O, Wagner M, Endres M, Villringer A, Röther J, Kolominsky-Rabas P, Berger K (2010): Schlaganfallhäufigkeit und Versorgung von Schlaganfallpatienten in Deutschland. Akt Neurol $\underline{37}, 333-340$ 
Hillen T, Coshall C, Tilling K, Rudd AG, McGovern R, Wolfe CDA (2003): Cause of stroke recurrence is multifactorial: patterns, risk factors, and outcomes of stroke recurrence in the South London Stroke Register. Stroke 34, 1457-1463

Hohnloser SH, Pajitnev D, Pogue J, Healey JS, Pfeffer MA, Yusuf S, Connolly SJ (2007): Incidence of stroke in paroxysmal versus sustained atrial fibrillation in patients taking oral anticoagulation or combined antiplatelet therapy: an ACTIVE W Substudy. J Am Coll Cardiol 50, 2156-2161

Homma S, Messé SR, Rundek T, Sun YP, Franke J, Davidson K, Sievert H, Sacco RL, Di Tullio MR (2016): Patent foramen ovale. Nat Rev Dis Primers $\underline{2}, 15086$

Iwasaki Y-k, Nishida K, Kato T, Nattel S (2011): Atrial fibrillation pathophysiology: implications for management: Implications for management. Circulation $\underline{124}$ 2264-2274

January CT, Wann LS, Calkins H, Chen LY, Cigarroa JE, Cleveland JC, Ellinor PT, Ezekowitz MD, Field ME, Furie KL et al. (2019): 2019 AHA/ACC/HRS Focused Update of the 2014 AHA/ACC/HRS guideline for the management of patients with atrial fibrillation: A report of the American College of Cardiology/American Heart Association Task Force on clinical practice guidelines and the Heart Rhythm Society. J Am Coll Cardiol 74, 104-132

Kamel H, Longstreth WT, Tirschwell DL, Kronmal RA, Broderick JP, Palesch YY, Meinzer C, Dillon C, Ewing I, Spilker JA et al. (2019): The atrial cardiopathy and antithrombotic drugs in prevention after cryptogenic stroke randomized trial: Rationale and methods. Int J Stroke 14, 207-214

Katsanos AH, Bhole R, Frogoudaki A, Giannopoulos S, Goyal N, Vrettou AR, Ikonomidis I, Paraskevaidis I, Pappas K, Parissis J et al. (2016): The value of transesophageal echocardiography for embolic strokes of undetermined source. Neurology $\underline{87}$, 988-995

Kernan WN, Ovbiagele B, Black HR, Bravata DM, Chimowitz MI, Ezekowitz MD, Fang MC, Fisher M, Furie KL, Heck DV et al. (2014): Guidelines for the prevention of stroke in patients with stroke and transient ischemic attack: a guideline for healthcare professionals from the American Heart Association/American Stroke Association. Stroke 45, 2160-2236

Kirchhof P, Benussi S, Kotecha D, Ahlsson A, Atar D, Casadei B, Castella M, Diener H-C, Heidbuchel $\mathrm{H}$, Hendriks J et al. (2016): 2016 ESC Guidelines for the management of atrial fibrillation developed in collaboration with EACTS. Eur Heart J 37, 2893-2962

Knebel F, Masuhr F, Hausen W von, Walde T, Dreger H, Raab V, Yuerek M, Baumann G, Borges AC (2009): Transesophageal echocardiography in patients with cryptogenic cerebral ischemia.

Cardiovasc Ultrasound $\underline{7}, 15$

Kolominsky-Rabas PL, Sarti C, Heuschmann PU, Graf C, Siemonsen S, Neundoerfer B, Katalinic A, Lang E, Gassmann KG, Stockert TR von (1998): A prospective community-based study of stroke in Germany-the Erlangen Stroke Project (ESPro): incidence and case fatality at 1, 3, and 12 months. Stroke 29, 2501-2506

Kolominsky-Rabas PL, Weber M, Gefeller O, Neundoerfer B, Heuschmann PU (2001):

Epidemiology of ischemic stroke subtypes according to TOAST criteria: incidence, recurrence, and long-term survival in ischemic stroke subtypes: a population-based study. Stroke 32, 2735-2740

Lambertz H, Lethen H: Transösophageale Echokardiographie, 2. Auflage; Georg Thieme Verlag, Stuttgart 2007 
Lambertz $\mathrm{H}$, Lethen $\mathrm{H}$ : Transösophageale Echokardiografie: Lehrbuch und Atlas zur Untersuchungstechnik und Befundinterpretation, 3. Auflage; Thieme, Stuttgart 2013

Laufs U, Hoppe UC, Rosenkranz S, Kirchhof P, Böhm M, Diener HC, Endres M, Grond M, Hacke W, Meinertz T et al. (2010): Cardiological evaluation after cerebral ischaemia Consensus statement of the Working Group Heart and Brain of the German Cardiac Society-Cardiovascular Research (DGK) and the German Stroke Society (DSG): Consensus statement of the Working Group Heart and Brain of the German Cardiac Society-Cardiovascular Research (DGK) and the German Stroke Society (DSG). Clin Res Cardiol 99, 609-625

Lechat P, Mas JL, Lascault G, Loron P, Theard M, Klimczac M, Drobinski G, Thomas D, Grosgogeat Y (1988): Prevalence of patent foramen ovale in patients with stroke. N Engl J Med $\underline{318}, 1148-1152$

Leung DY, Black IW, Cranney GB, Walsh WF, Grimm RA, Stewart WJ, Thomas JD (1995): Selection of patients for transesophageal echocardiography after stroke and systemic embolic events. Role of transthoracic echocardiography. Stroke $\underline{26}, 1820-1824$

Lusis AJ (2000): Atherosclerosis. Nature 407, 233-241

Lyden P (2017): Using the National Institutes of Health Stroke Scale: A Cautionary Tale. Stroke $\underline{48}$ 513-519

Mas JL, Derumeaux G, Amarenco P, Arquizan C, Aubry P, Barthelet M, Bertrand B, Brochet E, Cabanes L, Donal E et al. (2016): close: Closure of patent foramen ovale, oral anticoagulants or antiplatelet therapy to prevent stroke recurrence: Study design. Int J Stroke 11, 724-732

Mas JL, Derumeaux G, Guillon B, Massardier E, Hosseini H, Mechtouff L, Arquizan C, Béjot Y, Vuillier F, Detante $O$ et al. (2017): Patent foramen ovale closure or anticoagulation vs. antiplatelets after stroke. N Engl J Med $\underline{377}, 1011-1021$

Mayer F, Stahrenberg R, Gröschel K, Mostardt S, Biermann J, Edelmann F, Liman J, Wasem J, Goehler A, Wachter R et al. (2013): Cost-effectiveness of 7-day-Holter monitoring alone or in combination with transthoracic echocardiography in patients with cerebral ischemia. Clin Res Cardiol 102, 875-884

McGrath ER, Paikin JS, Motlagh B, Salehian O, Kapral MK, O'Donnell MJ (2014): Transesophageal echocardiography in patients with cryptogenic ischemic stroke: a systematic review. Am Heart J 168, 706-712

Meenan RT, Saha S, Chou R, Swarztrauber K, Pyle Krages K, O'Keeffe-Rosetti MC, McDonagh M, Chan BKS, Hornbrook MC, Helfand M (2007): Cost-effectiveness of echocardiography to identify intracardiac thrombus among patients with first stroke or transient ischemic attack. Med Decis Making 27, 161-177

Meier B, Kalesan B, Mattle HP, Khattab AA, Hildick-Smith D, Dudek D, Andersen G, Ibrahim R, Schuler G, Walton AS et al. (2013): Percutaneous closure of patent foramen ovale in cryptogenic embolism. N Engl J Med 368, 1083-1091

Meissner I, Khandheria BK, Heit JA, Petty GW, Sheps SG, Schwartz GL, Whisnant JP, Wiebers DO, Covalt JL, Petterson TM et al. (2006): Patent foramen ovale: innocent or guilty? Evidence from a prospective population-based study. J Am Coll Cardiol $\underline{47}$, 440-445

Mendis S, Puska P, Norrving B, Organization WH: Global Atlas on Cardiovascular Disease Prevention and Control; World Health Organization, Geneva 2011 
Menon BK, Coulter JI, Bal S, Godzwon C, Weeks S, Hutchison S, Hill MD, Coutts SB (2014): Acute ischaemic stroke or transient ischaemic attack and the need for inpatient echocardiography. Postgrad Med J $\underline{90}$, 434-438

Morris JG, Duffis EJ, Fisher M (2009): Cardiac workup of ischemic stroke: can we improve our diagnostic yield? Stroke $\underline{40}, 2893-2898$

Nabavi DG, Ossenbrink M, Schinkel M, Koennecke HC, Hamann G, Busse O (2015): Aktualisierte Zertifizierungskriterien für regionale und überregionale Stroke-Units in Deutschland. Nervenarzt 86, 978-988

Pepi M, Evangelista A, Nihoyannopoulos P, Flachskampf FA, Athanassopoulos G, Colonna P, Habib G, Ringelstein EB, Sicari R, Zamorano JL et al. (2010): Recommendations for echocardiography use in the diagnosis and management of cardiac sources of embolism: European Association of Echocardiography (EAE) (a registered branch of the ESC). Eur J Echocardiogr 11, 461-476

Peterson GE, Brickner ME, Reimold SC (2003): Transesophageal echocardiography: clinical indications and applications. Circulation 107, 2398-2402

Ponikowski P, Voors AA, Anker SD, Bueno H, Cleland JGF, Coats AJS, Falk V, González-Juanatey JR, Harjola V-P, Jankowska EA et al. (2016): 2016 ESC Guidelines for the diagnosis and treatment of acute and chronic heart failure: The Task Force for the diagnosis and treatment of acute and chronic heart failure of the European Society of Cardiology (ESC)Developed with the special contribution of the Heart Failure Association (HFA) of the ESC. Eur Heart J 37, 2129-2200

Rauh R, Fischereder M, Spengel FA (1996): Transesophageal echocardiography in patients with focal cerebral ischemia of unknown cause. Stroke 27, 691-694

Ringelstein EB, Nabavi DG: Der ischämische Schlaganfall: Eine praxisorientierte Darstellung von Pathophysiologie, Diagnostik und Therapie, 1. Auflage; Kohlhammer Verlag, Stuttgart 2007

Ringleb, Bousser MG, Ford G, Bath P, Brainin M, Caso V, Cervera A, Chamorro A, Cordonnier C, Csiba $L$ et al. (2008): Guidelines for management of ischaemic stroke and transient ischaemic attack 2008. Cerebrovasc. Dis. $\underline{25}, 457-507$

Rus Mansilla C, Mesa Rubio D, Suarez de Lezo Cruz Conde J, Rodriguez Almodovar A, Duran Torralbo C, Delgado Ortega M (2008): Utility of transesophageal echocardiography in young patients with cryptogenic stroke and low cardiovascular risk. Med Clin (Barc) 130, 241-245

Sanna T, Diener HC, Passman RS, Di Lazzaro V, Bernstein RA, Morillo CA, Rymer MM, Thijs V, Rogers T, Beckers F et al. (2014): Cryptogenic stroke and underlying atrial fibrillation. N Engl J Med $\underline{370}, 2478-2486$

Sansoy V, Abbott RD, Jayaweera AR, Kaul S (1995): Low yield of transthoracic echocardiography for cardiac source of embolism. The American Journal of Cardiology 75, 166-169

Saver JL (2016): CLINICAL PRACTICE. Cryptogenic Stroke. N Engl J Med 374, 2065-2074

Sever PS, Dahlöf B, Poulter NR, Wedel H, Beevers G, Caulfield M, Collins R, Kjeldsen SE, Kristinsson A, Mclnnes GT et al. (2003): Prevention of coronary and stroke events with atorvastatin in hypertensive patients who have average or lower-than-average cholesterol concentrations, in the Anglo-Scandinavian Cardiac Outcomes Trial-Lipid Lowering Arm (ASCOT-LLA): a multicentre randomised controlled trial. The Lancet $\underline{361}, 1149-1158$ 
Sörös P, Hachinski V (2012): Cardiovascular and neurological causes of sudden death after ischaemic stroke. The Lancet Neurology 11, 179-188

Stokman PJ, Nandra CS, Asinger RW (2001): Left ventricular thrombus. Curr Treat Options Cardio Med $\underline{3}, 515-521$

Strandberg M, Marttila RJ, Helenius H, Hartiala J (2002): Transoesophageal echocardiography in selecting patients for anticoagulation after ischaemic stroke or transient ischaemic attack. J. Neurol. Neurosurg. Psychiatr. $\underline{73}, 29-33$

Ustrell X, Pellisé A (2010): Cardiac workup of ischemic stroke. Curr Cardiol Rev $\underline{6}, 175-183$ Vahanian A, Alfieri O, Andreotti F, Antunes MJ, Baron-Esquivias G, Baumgartner H, Borger MA, Carrel TP, Bonis M de, Evangelista A et al. (2012): Guidelines on the management of valvular heart disease (version 2012). Eur Heart J 33, 2451-2496

Vaitkus PT, Barnathan ES (1993): Embolic potential, prevention and management of mural thrombus complicating anterior myocardial infarction: A meta-analysis. J Am Coll Cardiol $\underline{22}$, 1004-1009

van den Bussche H, Berger K, Kemper C, Barzel A, Glaeske G, Koller D (2010): Inzidenz, Rezidiv, Pflegebedürftigkeit und Mortalität von Schlaganfall. Akt Neurol 37, 131-135

van Swieten., Koudstaal PJ, Visser MC, Schouten HJ, van Gijn J (1988): Interobserver agreement for the assessment of handicap in stroke patients. Stroke $\underline{19}, 604-607$

Vanassche T, Lauw MN, Eikelboom JW, Healey JS, Hart RG, Alings M, Avezum A, Díaz R, Hohnloser $\mathrm{SH}$, Lewis BS et al. (2015): Risk of ischaemic stroke according to pattern of atrial fibrillation: analysis of 6563 aspirin-treated patients in ACTIVE-A and AVERROES. Eur Heart J $\underline{36}, 281-7 a$

Wachter R, Gröschel K, Gelbrich G, Hamann GF, Kermer P, Liman J, Seegers J, Wasser K, Schulte A, Jürries $F$ et al. (2017): Holter-electrocardiogram-monitoring in patients with acute ischaemic stroke (Find-AF RANDOMISED ): an open-label randomised controlled trial: An open-label randomised controlled trial. The Lancet Neurology 16, 282-290

Wachter R, Stahrenberg R, Gröschel K (2013): Subclinical atrial fibrillation: how hard should we look? Heart $\underline{99}, 151-153$

Wallmann D, Tuller D, Wustmann K, Meier P, Isenegger J, Arnold M, Mattle HP, Delacretaz E (2007): Frequent atrial premature beats predict paroxysmal atrial fibrillation in stroke patients: an opportunity for a new diagnostic strategy. Stroke 38, 2292-2294

Ward A, Payne KA, Caro JJ, Heuschmann PU, Kolominsky-Rabas PL (2005): Care needs and economic consequences after acute ischemic stroke: the Erlangen Stroke Project. European Journal of Neurology $\underline{12}$

Warner MF (1996): Routine Transesophageal Echocardiography for Cerebral Ischemia. Arch Intern Med $\underline{156}, 1719$

Wasser K, Weber-Krüger M, Jürries F, Liman J, Hamann GF, Kermer P, Uphaus T, Protsenko E, Seegers J, Mende M et al. (2019): The cardiac diagnostic work-up in stroke patients-A subanalysis of the Find-AFRANDOMISED trial. PLoS ONE 14, e0216530

Weber-Krüger M, Gelbrich G, Stahrenberg R, Liman J, Kermer P, Hamann GF, Seegers J, Gröschel K, Wachter R (2014): Finding atrial fibrillation in stroke patients: Randomized evaluation of 
enhanced and prolonged Holter monitoring--Find-AF(RANDOMISED) - rationale and design. Am Heart J $\underline{168}, 438-445 . e 1$

Wechsler LR (2008): PFO and stroke: What are the data? Cardiol Rev $\underline{16}, 53-57$

World Medical Association (2013): World Medical Association Declaration of Helsinki: ethical principles for medical research involving human subjects: Ethical principles for medical research involving human subjects. JAMA $\underline{310}, 2191-2194$

Yang H, Nassif M, Khairy P, Groot JR de, Roos YBWEM, Winter RJ de, Mulder BJM, Bouma BJ (2016): Cardiac diagnostic work-up of ischaemic stroke. Eur Heart J

Zeymer U, Kastrati A, Rassaf T, Scholz K-H, Thiele H, Nienaber CA (2013): Kommentar zu den Leitlinien der Europäischen Gesellschaft für Kardiologie (ESC) zur Therapie des akuten Herzinfarkts bei Patienten mit ST-Streckenhebung (STEMI). Kardiologe $\underline{7}$, 410-422 


\section{Danksagung}

Zunächst möchte ich mich bei Herrn Prof. Dr. med. Rolf Wachter als Doktorvater für die Überlassung des Themas und Begleitung während der Anfertigung der Dissertationsschrift bedanken. Die Zusammenarbeit in der durch ihn geleiteten Arbeitsgruppe war stets geprägt durch hohe fachliche Kompetenz, fruchtbare Gespräche und eine harmonische Zusammenarbeit.

Ebenso möchte ich auch Herrn Dr. med. Mark Weber-Krüger als direktem Betreuer dieser Arbeit danken. Der hohe Einsatz und angenehme persönliche Kontakt, der über das übliche Maß hinausging, hat zusammen mit seinen Anregungen und seiner Motivation in schweren Zeiten maßgeblich zur Fertigstellung dieser Arbeit beigetragen.

Weiterhin gilt mein Dank der Klinik für Kardiologie und Pneumologie (Direktor Prof. Dr. Gerd Hasenfuß) der Universitätsmedizin Göttingen für die Möglichkeit, meine Promotion in dieser Abteilung durchführen zu können.

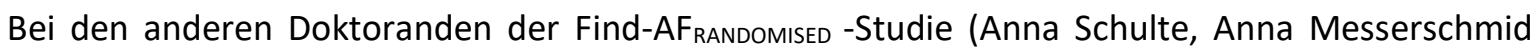
und Nico Behnke) möchte ich mich ebenfalls für die kollegiale und enge Zusammenarbeit bedanken. 